\title{
Printed photodetectors
}

\author{
Giuseppina Pace $^{1}$, Andrea Grimoldi ${ }^{1,2}$, Marco Sampietro ${ }^{1,2}$, Dario Natali ${ }^{1,2}$ and Mario Caironi ${ }^{1}$ \\ ${ }^{1}$ Center for Nano Science and Technology@PoliMi, Istituto Italiano di Tecnologia, Via Pascoli 70/3, \\ I-20133 Milano, Italy \\ ${ }^{2}$ Dipartimento di Elettronica, Informazione e Bioingegneria, Politecnico di Milano, P.za L. Da Vinci, 32 \\ I-20133 Milano, Italy \\ E-mail: dario.natali@polimi.it and mario.caironi@iit.it
}

\begin{abstract}
Photodetectors convert light pulses into electrical signals and are fundamental building blocks for any opto-electronic system adopting light as a probe or information carrier. They have widespread technological applications, from telecommunications to sensors in industrial, medical and civil environments. Further opportunities are plastic short-range communications systems, interactive large-area surfaces and light-weight, flexible, digital imagers. These applications would greatly benefit from the cost-effective fabrication processes enabled by printing technology. While organic semiconductors are the most investigated materials for printed photodetectors, and are the main focus of the present review, there are notable examples of other inorganic or hybrid printable semiconductors for opto-electronic systems, such as quantum-dots and nanowires. Here we propose an overview on printed photodetectors, including three-terminal phototransistors. We first give a brief account of the working mechanism of these light sensitive devices, and then we review the recent progress achieved with scalable printing techniques such as screen-printing, inkjet and other non-contact technologies in the development of all-printed or hybrid systems.
\end{abstract}

Keywords: printed photodetectors, organic semiconductors, optoelectronics

\section{Introduction}

Light can either carry a large amount of information when modulated in an electro-optical or photonic circuit, or can reveal specific features of a medium when used as a probe. In both cases, since information handling and storage is so far best accomplished by means of electronic computing, light has to be transduced into an electronic signal. This task is performed by a photodetector (PD). The specific applications of a photodetector vary according to the required sensitivity to light intensity, to the spectral response and to the speed response. The strong impact of photodetectors into nowadays technology can be easily deduced from their wide exploitation in our daily life. Besides being a fundamental building block for light-based telecommunication systems, they can be found in automated building gates and doors, light switches, DVD, blu-ray devices, or in the remote controls of our domestic appliances for instance. The advanced use of light sensors further extends to a vast range of applications in information processing, photonic integrated systems, x-ray medical imaging and spectroscopy, alignment systems, position detection, industrial manufacturing, time and frequency measurements, short range plastic-fiber based transceivers, integrated sensors for Lab-on-a-chip reactors. The advent of complementary metal oxides semiconductors (CMOS) has enabled the development of image sensors fostering the growth of the rich market for digital cameras and other image sensors and scanners.

So far, the technology development in this field has mostly targeted the device miniaturization, the achievement of an increased responsivity, short time response, low noise and operational voltage and high dynamic range. Inorganic compounds have been playing a major role as active materials for light detection. Silicon is the most exploited one, enabling light detection in a wide spectral range going from the visible to the near-infrared (NIR), up to $1.1 \mu \mathrm{m}$. A main limit in 
silicon detectors technology is due to the relatively low absorption coefficient (e.g. silicon reaches $10^{4} \mathrm{~cm}^{-1}$ at 500 $\mathrm{nm}$ ) imposing device thicknesses of the order of several microns to reduce transmission losses. For the light detection in the IR region, a quantum efficiency higher than $80 \%$ is achieved in GaAs PDs at $840 \mathrm{~nm}$, only if the film thickness is higher than $1.8 \mu \mathrm{m}$ [1]. One of the main strategies employed so far to enhance photon absorption in PDs is the nanostructuring of the device layers to create waveguiding effects. The latter approach limits the device downscaling, adding complexity to the fabrication process and resulting in an increasing cost for the integrations of those nanopatterned structures into the large scale production of optoelectronic devices [2].

As an alternative to high vacuum and high temperature techniques so far employed for inorganic materials deposition, solution based strategies, which mainly exploit organic semiconductors and more recently $1 \mathrm{D}$ and 2D materials, as graphene, quantum dots (QDs) and carbon nanotubes, have been developed [3]. The formulation of materials into functional inks allows the fabrication of optoelectronic devices via printing techniques. This enables device fabrication scalability, integration with electronic components at lower production costs and opens the way to large-area applications. Among solution-processable semiconductors, organic conjugated materials quite easily lend themselves to printing, thanks to the possibility of tuning the formulations rheology and therefore of engineering inks for different printing techniques and applications [2, 4-6]. Their low-temperature processing $\left(<150{ }^{\circ} \mathrm{C}\right)$ allows the device fabrication on a vast range of substrates, rigid and flat as well as flexible and curved, opening the way for the development of non-fragile, conformable electronics.

Not only organic semiconductors allow solution processability, but their chemical tunability also enables high selectivity over a large spectral range, which goes from the UV-vis to the NIR [7]. By a proper design of their molecular structure and thin-film packing motif, the optical bandgap and electronic transport properties can be tuned. Highly selective compounds with very narrow spectral response, as well as broad band absorbing materials can be synthesized [8-10]. Nevertheless, charge carrier mobility in organic semiconductors is generally lower than their inorganic counterparts, though recently improved mobility values $\left(\sim 10^{-3} \mathrm{~cm}^{2}\right.$ $\mathrm{V}^{-1} \mathrm{~s}^{-1}$ for bulk transport at low charge density) have been obtained [11]. Importantly, organic semiconductors ensure longer carrier lifetime and strong absorption coeffi-cient (up to $10^{5} \mathrm{~cm}^{-1}$ ) with respect to inorganic semi-conductors [12]. Such strong absorption enables the use of very thin films $(<100 \mathrm{~nm}$ thick), so that carrier transit times can be low despite the limited carrier mobility [13]. It has already been demonstrated that organic photodetectors can operate with bandwidths exciding $10 \mathrm{MHz}$ up to $430 \mathrm{MHz}$ [14-19].

Hybrid technology, which combines organic and inorganic semiconductors in particular nanowires and nanoparticles, is also very promising [20]. Solution processable inorganic nanoparticles have extended the operational window in terms of spectral response and the possibility to be operated in ambient conditions. For a review of the current studies on solution processable inorganic photodetectors the reader is addressed to previous reports [21]. The electronic structure of inorganic nanoparticles is strongly dependent on their dimension and shape. For a low number of atoms present in the nanoparticle cluster quantized states are observed due to particle size confinement effect. However, their coales-cence into large clusters at the solid state has the main effect of removing this quantization, and conduction and valence bands start being observed [22]. QDs can be flexibly tailored in composition and size, offering a wide spectral response. Moreover they can be easily synthesized and deposited using high-throughput processes such as inkjet printing [23] and spray coating [24]. Current NIR technologies rely on the epitaxial growth of film of inorganic semiconductors onto rigid substrates. QDs are so far among these solution processable semiconductors, that can allow light detection into the IR region up to $3 \mu \mathrm{m}$ [25] and even $5 \mu \mathrm{m}$ [26]. This can further foster the development in applications like passive night vision, safety sensors, bio-diagnostic and optical communications [3].

An increasing number of printable organic semiconductors and nanoparticles employed as active materials in photodetectors are being proposed in the literature, and this flourishing field is speeding toward scalability and industrial commercialization.

In this report, we will review the most recent works on printed photodetectors, keeping our focus on the solution processing methods applied for the devices fabrication and their scaling up. We will highlight the recent examples of printed organic photodetectors integrated into electronic arrays, this being the first step towards an industrial scaled-up manufacturing of printed opto-electronics.

In section 2 we describe the working principles of photodetectors and phototransistors based on solution processable organic and inorganic materials. In section 3 we report on the main printing methods employed for photodetectors fabrication: their implementation, experimental challenges, limits and advantages are commented on the basis of recent relevant devices and systems proposed in the literature.

\section{Photodetectors and phototransistors: basic working mechanism and relevant figure of merits}

Among the diverse light detecting devices and concepts which have been developed over decades of research [27], the most widely exploited scheme is the one where light absorption leads to the generation of separated holes and electrons, which are subsequently collected to opposite electrodes. Such mechanism is typical of inorganic $p-n$ junctions as also of donor-acceptor organic semiconductors.

The basic processes leading to the charge excitation, dissociation and migration to the electrodes are intrinsically dependent on the nature of the active materials. The physics behind these processes in organic materials has been subject of deep studies in the past and comprehensive reviews have 
(a)

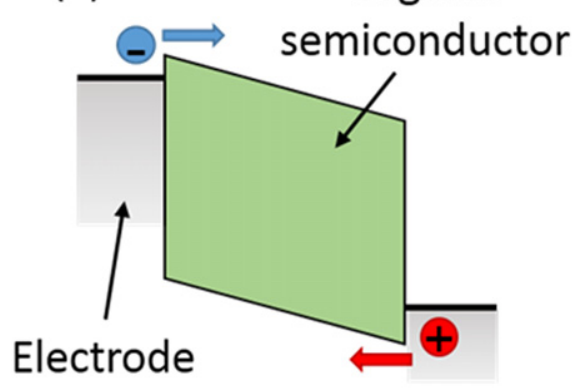

(b)

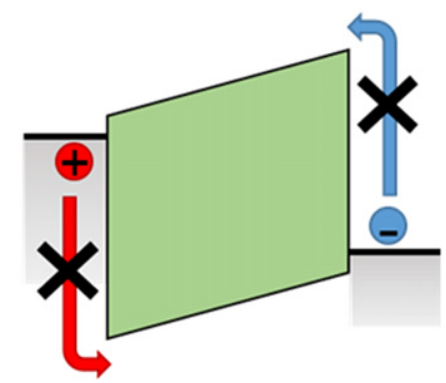

(c)

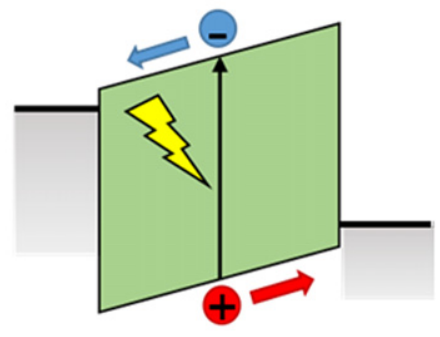

Scheme 1. Scheme of charge injections from electrodes to semiconductor in direct bias (a) and reverse bias (b). (c) Photogenerated charges are separated and transported to the electrodes thanks to the electric field generated by the reverse bias applied to the detector.

already been reported [21, 28]. A simple sketch of the electronic structure of organic semiconductors can be drawn by knowing the frontier $\pi$-orbitals of an isolated molecule, the highest occupied molecular orbital (HOMO) and lowest unoccupied molecular orbital (LUMO). A discrete broadening around those levels exists in the solid-state and it is mostly originated by morphological disorder. Importantly, the primary photo-excitation product is typically represented by a strongly bound $(0.1-0.5 \mathrm{eV})$ molecular exciton, i.e. a highly localized and neutral state, which at room temperature does not dissociate efficiently into free charges [29].

The main strategy employed to foster efficient charge dissociation in organic semiconductors has been to intermix donor (D) and acceptor (A) materials in solution, followed by their deposition into films where bulk D-A heterojunctions are formed. By properly engineering the HOMO and LUMO levels of both $\mathrm{D}$ and $\mathrm{A}$, excitons which are either photo-generated next to a $\mathrm{D} / \mathrm{A}$ interface or have diffused there, can dissociate with high yield into electrons and holes. The driving force for charge dissociation is given by the difference between the electron affinity of the excited D and the electron affinity of the A, minus the coulombic attraction. This latter is a non-negligible barrier to be accounted for, given the low dielectric constant of organic materials $\varepsilon_{\mathrm{r}}=3-4$, which limits the dissociation yield of excitons. However, differently from solar cells, in photodetectors exciton dissociation into free charges and their subsequent transport to the electrodes, can be further promoted by the electric field introduced by an external applied bias. It has to be noted that an external bias results in a current flow due to the injection of carriers from the contacts even in absence of light. This current, termed dark current, can be minimized by properly selecting the work-function of the contacts. According to the bias polarity a forward and a reverse bias can be distinguished. In forward bias, a current can be easily injected and typically follows a space-charge limited behavior. In reverse bias, suitable injection barriers strongly limit carrier injection in the active material (scheme 1) [30].

The path followed by a photogenerated charge is different in photodiodes and in photoconductors. In photodiodes excess carriers travel within the two active D and A phases directed towards their respective electrodes were they are finally collected: the holes at the anode and the electrons at the cathode. In photoconductors, one of the two charge carriers, either holes or electrons, is trapped into localized states. This carrier is therefore mostly unavailable for the charge transport, while the opposite carrier is mobile and, once collected, is re-injected at the opposite electrode-provided that contacts are ohmic - in order to maintain charge neutrality.

Although planar structures avoid the need for a transparent electrode and can be easier to realize than the vertical ones, they present a much larger distance between the electrodes $(L)$ which is typically in the range of few to tens of micrometers. Such length can drastically increase the carrier transit time, and its downscaling would require high resolution lithographic processes. Scaling of $L$ is instead more easily achieved in a vertical structure, where the distance between the electrodes is defined by the thickness of a solution pro-cessed active layer, in the range from few tens to few hun-dreds of nanometers, providing much faster collection of charges. Both structures can either operate as a photodiode or a photoconductor.

In addition to two-terminal devices, also three terminal devices such as field effect transistors (FETs) can be employed as light sensors, typically with the scope of achieving very high responsivity. They are termed photo-transistors since the channel conductance can be modulated by photogenerated charges in addition to the field effect exerted by the gate. Their working mechanism has been already described elsewhere [21, 28].

Some figures of merit have to be taken into account in order to characterize and compare photodetectors. Here they are concisely highlighted, for a more detailed dissertation we refer to text books [31, 32].

The conversion efficiency of impinging photons to photocurrent is measured by the external quantum efficiency (EQE) of the detector, given by the ratio between the number of charges collected per second at the electrodes and the number of incident photons per second. Excluding ionizing radiation detectors and avalanche multiplying mechanisms, in photodiodes the maximum quantum efficiency achievable is $100 \%$, since per each photon absorbed the yield of holeelectron charge generation and dissociation cannot be higher than 1. For what concerns ionizing radiation detectors, one must take into account that more than one electron-hole pair can be generated per quantum of radiation. In the 
photoconductive regime more than one charge per photon can flow through the device so that efficiencies can be higher than $100 \%$ according to the ratio between the lifetime of the trapped charge and the transit time of the mobile one.

Responsivity $\left(R, \mathrm{~A} \mathrm{~W}^{-1}\right)$ can be used alternatively to the $\mathrm{EQE}$, the relation among the two being:

$$
R=\frac{\mathrm{EQE} \cdot \lambda \cdot q}{h c},
$$

where $\lambda$ is the wavelength of interest, $h$ the Planck constant, $c$ the speed of light, $q$ the elementary charge. $R$ indicates the photocurrent which can be extracted per Watt of impinging radiation, at a specific wavelength. With respect to a plot of $\mathrm{EQE}$ versus $\lambda$, the spectral shape of $R$ versus $\lambda$ thus tends to favor longer wavelengths, where more photons compose the incoming signal at a specific power.

Another important figure of merit to be considered in photodetectors is the ratio between the signal power and the noise power, termed signal to noise ratio (SNR). In order to calculate SNR the main sources of noise must be taken into account, and in particular the dark current shot noise and the photocurrent noise, which lead to a noise spectral density $\left(S_{\mathrm{n}}\right)$

$$
S_{\mathrm{n}}=2 q\left(I_{\mathrm{dk}}+I_{\mathrm{ph}}\right),
$$

where $I_{\mathrm{dk}}$ is the dark current and $I_{\mathrm{ph}}$ the photocurrent. Even if the photocurrent is lower than the dark current, a signal can still be detected provided that the root mean square of the fluctuation due to the noise is lower than the signal value. In this case dark current shot noise is dominant with respect to the photocurrent one, making the latter negligible. Also a frequency dependent noise component, namely flicker noise, is typically present. The flicker noise has a power spectral density (energy per power per $\mathrm{Hz}$ ) which is proportional to the inverse of the frequency of the photodiode signal. In this case the total amount of noise should be carefully measured in order not to underestimate its value.

Specific detectivity $\left(D^{*}\right)$ is usually adopted to express the capability of the device to detect small signals and it is proportional to the ratio between the responsivity and the noise spectral density. When the dark current shot noise is dominant, $D^{*}$ can be written as:

$$
D^{*}=\frac{J_{\mathrm{ph}}}{I_{\mathrm{opt}} \sqrt{2 q J_{\mathrm{dk}}}},
$$

where $I_{\mathrm{opt}}$ is the impinging optical power density and $J_{\mathrm{ph}}$ and $J_{\mathrm{dk}}$ are respectively the photocurrent density and dark current density. Keeping constant the responsivity (i.e. the ratio $\left.J_{\mathrm{ph}} / I_{\mathrm{opt}}\right)$ a photodetector with lower dark current density is able to detect smaller light signals and it has a larger $D^{*}$. Photodiodes are typically operated in reverse bias regime to limit dark currents and to improve the SNR. Silicon photodetectors are characterized by a $D^{*}$ above $10^{13}$ Jones in the spectral range between 600 and $1000 \mathrm{~nm}$.

In order to evaluate the photodetector speed, its response time is measured as the time in which the current, starting from the dark current value, reaches the steady value after the exposure to light. To be more specific, the rise (fall) time is defined as the time needed for the photocurrent to grow (decay) from $10 \%(90 \%)$ to the $90 \%(10 \%)$ of $\Delta I$, where $\Delta I$ is the difference between the steady state current upon a rectangular light stimulus and the dark current. In the frequency domain, the relevant figure of merit is the bandwidth, usually defined as the frequency at which the responsivity drops by $3 \mathrm{~dB}$ with respect to its low-frequency value.

Because for photoconductors the efficiency can be higher than $100 \%$, they are also characterized by a photoconductive gain $(G)$, defined as the ratio between the transit time of the flowing charge carrier $(\tau)$ and the recombination time $\left(\tau_{\mathbf{r}}\right)$

$$
G=\frac{\tau}{\tau_{\mathrm{r}}}
$$

Since the response time is proportional to the recombination time, there is a trade-off between the photoconductive gain and the response time.

When photodetectors are employed to detect x-rays photons, the energies of which span from tens of $\mathrm{keV}$ to $\mathrm{MeV}$, specific figures of merit exist. Performances of x-ray detectors are expressed in terms of sensitivity and detective quantum efficiency (DQE). The first one is defined as the charge produced by the detector per impinging $x$-ray quantum at a specified energy. The latter expresses the efficiency in transferring the signal to the output without adding noise to the one intrinsically associated to the Poisson distribution of the impinging quanta; high DQE values indicate that less radiation is needed to achieve the same output SNR. To evaluate $\mathrm{X}$-ray imagers response time, the image lag is often used. It is defined as the ratio between the signal read at a certain time delay after the end of x-ray exposure and the signal read when the imager is exposed to radiation. At a fixed delay time, the smaller is the lag, the faster is the response time.

In the case of phototransistors [33, 34], in addition to typical FETs figures of merits, such as mobility and threshold voltage, other figures of merit are used i.e., the photocurrent on/off ratio and responsivity $\left(R_{\mathrm{pt}}\right)$. The on/off ratio is defined as the ratio between drain current measured when the transistor is exposed to light and the one measured with the transistor kept into dark, while the responsivity of a phototransistor is defined as follows:

$$
R_{\mathrm{pt}}=\frac{J_{\mathrm{ph}}}{I_{\mathrm{opt}}}=\frac{J_{\mathrm{d}, \mathrm{ph}}-J_{\mathrm{d}, \mathrm{dk}}}{I_{\mathrm{opt}}},
$$

where $J_{\mathrm{d}, \mathrm{ph}}$ is the drain current density under illumination and $J_{\mathrm{d}, \mathrm{dk}}$ is the drain dark current density. A summary of the main figures of merit reported above are reported in table 1.

\section{State of the art of printed photodetectors}

In contrast to lithographic methods, mostly employed in the fabrication of inorganic devices via a subtractive approach, printing technology uses an additive processing which involves a single deposition step per layer, eventually followed by a drying or sintering step. In this review we will report only those printing techniques successfully employed for the fabrication of organic photodetectors as inkjet, 
Table 1. Figures of merit in photodetectors and phototransistors.

\begin{tabular}{|c|c|c|c|}
\hline Quantity & Symbol/Acronym & Unit & Definition/Physical meaning \\
\hline Dark current & $I_{\mathrm{dk}}$ & A & Current flowing in absence of illumination \\
\hline Photocurrent & $I_{\mathrm{ph}}$ & A & Current flowing under illumination \\
\hline $\begin{array}{l}\text { External quantum } \\
\text { efficiency }\end{array}$ & EQE & - & $\begin{array}{l}\text { Ratio between the number of collected charge } \\
\text { carriers and the number of incident photons } \\
\text { per second }\end{array}$ \\
\hline Responsivity & $R$ & $\mathrm{~A} \mathrm{~W}^{-1}$ & $\begin{array}{l}\text { Ratio between the photocurrent and the optical } \\
\text { power impinging on the device }\end{array}$ \\
\hline Rise (fall) time & $\tau$ & $\mathrm{s}$ & $\begin{array}{l}\text { Rise (fall) time is the time needed for the } \\
\text { photocurrent to grow(decay) from } 10 \% \\
\text { ( } 90 \% \text { ) to the } 90 \%(10 \%) \text { of } \Delta I \text {, where } \Delta I \text { is } \\
\text { the difference between the steady state cur- } \\
\text { rent upon a rectangular light stimulus and } \\
\text { the dark current. }\end{array}$ \\
\hline Bandwidth & BW & $\mathrm{Hz}$ & $\begin{array}{l}\text { Frequency at which responsivity drops by } 3 \mathrm{~dB} \\
\text { with respect to low frequency value }\end{array}$ \\
\hline Signal to noise ratio & SNR & - & $\begin{array}{l}\text { Power ratio between the signal and the noise of } \\
\text { the detector }\end{array}$ \\
\hline \multirow[t]{2}{*}{ Specific detectivity } & $D^{*}$ & $\frac{\mathrm{cm} \sqrt{\mathrm{Hz}}}{\mathrm{W}}$ (Jones) & \\
\hline & & & $\begin{array}{l}\text { Measure of the capability of the device to } \\
\text { detect small signals. It is used to compare } \\
\text { sensitivity of different detectors }\end{array}$ \\
\hline Photoconductive gain & $G$ & - & $\begin{array}{l}\text { Ratio between transit time of the flowing } \\
\text { charge carrier and recombination time }\end{array}$ \\
\hline Photransistor on/off ratio & On/Off & - & $\begin{array}{l}\text { Ratio between drain current measured when } \\
\text { the transistor is exposed to light and the one } \\
\text { measured with the transistor kept into dark }\end{array}$ \\
\hline $\begin{array}{l}\text { Phototransistor } \\
\text { responsivity }\end{array}$ & $R_{\mathrm{pt}}$ & $\mathrm{A} \mathrm{W}^{-1}$ & $\begin{array}{l}\text { Ratio between the variation of current in the } \\
\text { channel upon illumination and impinging } \\
\text { optical power }\end{array}$ \\
\hline
\end{tabular}

Figure of merit for $\mathrm{x}$-ray detectors and imagers

\begin{tabular}{|c|c|c|c|c|}
\hline Sensitivity & $S$ & $\frac{\mathrm{C}}{\mathrm{R} \mathrm{cm}^{2}}$ & $\mathrm{R}=($ Roentgen $)$ & $\begin{array}{l}\text { The charge produced by the detector per } \\
\text { impinging x-ray quantum at a specified } \\
\text { energy }\end{array}$ \\
\hline $\begin{array}{l}\text { Detective quantum } \\
\text { efficiency }\end{array}$ & DQE & - & & $\begin{array}{l}\text { Expresses the efficiency in transferring the } \\
\text { signal to the output without adding noise to } \\
\text { the one intrinsically associated to the Pois- } \\
\text { son distribution of the impinging quanta }\end{array}$ \\
\hline
\end{tabular}

spraying and screen printing, which are presented in the following section. Table 2 reports the electro-optical characteristics of the most significant printed OPD considered in this review.

\subsection{Inkjet printed photodetectors and phototransistors}

Inkjet printing is based on a drop-by-drop deposition approach [43]. The droplets of a low viscosity solution are jetted by a nozzle and can be generated either continuously or on demand (drop-on-demand, DOD). In the continuous mode a pressure, owing to Rayleigh instability (scheme 2), ejects ink from the nozzle creating a stream of liquid that forms droplets that are charged by an electric field applied to the nozzle. Charged droplets are separated from the uncharged ones and deflected by an electric field before reaching the substrate. The uncharged droplets are undeflected and collected by a gutter and re-used. The very high ejecting frequency (65000-165000 droplets $\mathrm{s}^{-1}$ ) enables fast printing. Nozzle clogging is prevented because the jet is always active, allowing also the use of volatile solvents. However, for desktop applications, industrial productions requiring a higher precision and lab-scale research, the DOD system is more commonly used because of a lower system complexity and 
Table 2. Summary of the best performances achieved for printed photodetectors.

\begin{tabular}{|c|c|c|c|c|c|c|c|c|}
\hline $\begin{array}{l}\text { Printing } \\
\text { method }\end{array}$ & $\begin{array}{c}\text { Device } \\
\text { structure }\end{array}$ & $\begin{array}{l}\text { Active } \\
\text { material }\end{array}$ & $\begin{array}{c}D^{*} \\
\text { (Jones) }\end{array}$ & $\begin{array}{c}R \\
\left(\mathrm{~A} \mathrm{~W}^{-1}\right)\end{array}$ & $\begin{array}{c}\tau \\
(\mathrm{ms})\end{array}$ & $\begin{array}{c}\text { Bias } \\
\text { voltage } \\
\text { (s) }(\mathrm{V})\end{array}$ & $\begin{array}{c}\text { Lateral } \\
\text { dimensions } \\
(\mu \mathrm{m} \times \mu \mathrm{m})\end{array}$ & References \\
\hline Inkjet & Photodetector & Polymer & $1.4 \times 10^{13}$ & 0.25 & - & 0.1 & $4000 \times 4000$ & [35] \\
\hline Inkjet & Photodetector & Polymer & $1.5 \times 10^{12}$ & 0.39 & 0.1 & 1 & $100 \times 90$ & [36] \\
\hline Inkjet & Phototransistor & $\begin{array}{c}\text { Small } \\
\text { molecule }\end{array}$ & - & $10^{6}$ & $10^{3}$ & $\begin{array}{c}V_{\mathrm{G}}=20 \\
V_{\mathrm{DS}}=20\end{array}$ & $500 \times 0.7$ & {$[37]$} \\
\hline Inkjet & Phototransistor & Polymer & - & 0.11 & - & $\begin{array}{c}V_{\mathrm{G}}=-10 \\
V_{\mathrm{DS}}=1\end{array}$ & $125 \times 10$ & [38] \\
\hline Spray & Photodetector & $\begin{array}{l}\text { Quantum } \\
\text { dots }\end{array}$ & $\begin{array}{c}1 \times 10^{10} \\
(800-1000) \mathrm{nm}\end{array}$ & $\begin{array}{c}0.5 \\
(800-1100) \mathrm{nm}\end{array}$ & 2 & & $15000 \times 3$ & [39] \\
\hline Spray & Photodetector & Polymer & $\begin{array}{c}8.8 \times 10^{11} \\
(532 \mathrm{~nm})\end{array}$ & $\begin{array}{c}0.36 \\
(532 \mathrm{~nm})\end{array}$ & - & $-5 \mathrm{~V}$ & $2000 \times 2000$ & [40] \\
\hline Spray & Photodetector & $\begin{array}{l}\text { Metal } \\
\text { oxide }\end{array}$ & - & $\begin{array}{c}622 \\
(350 \mathrm{~nm})\end{array}$ & $1.8 \times 10^{3}$ & $5 \mathrm{~V}$ & $2500 \times 200$ & [41] \\
\hline Spray & Photodetector & Polymer & - & $\begin{array}{c}0.01 \\
(660 \mathrm{~nm})\end{array}$ & $10^{-2}$ & $1 \mathrm{~V}$ & - & [42] \\
\hline
\end{tabular}

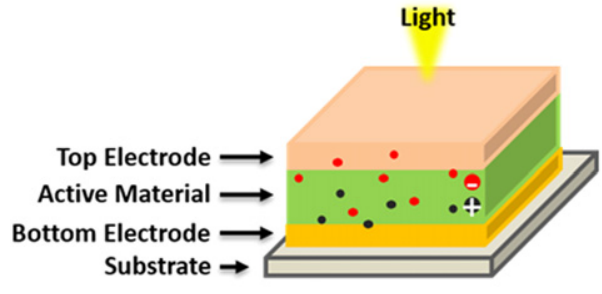

a)

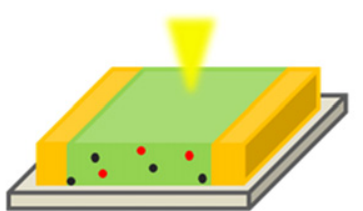

b)

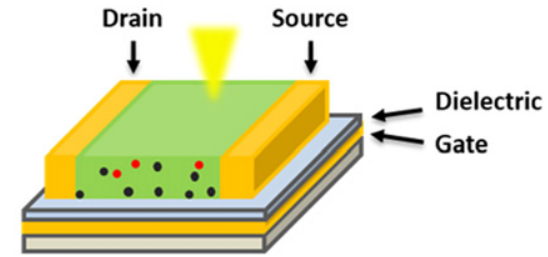

c)

Scheme 2. Sketches of the most common photodetectors structures: (a) vertical and (b) planar architectures. With the addition of a gate electrode and oxide, the planar structure can be turned into a three terminal photo-transistor device (c).

higher accuracy of droplet displacement. With DOD, thermal bubble techniques or piezoelectric transducers are used to force droplets out of the nozzle (scheme 3). A thermal printhead uses a heater causing a rapid vaporization of the ink and inducing the formation of a bubble. Consequently, the pressure in the nozzle increases, forcing a droplet to be ejected. Thermal DOD technology is simple in design and features lower costs, but it is restricted to vaporizable fluids required for the bubble's formation. Due to this limitation, piezoelectric transduction is commonly preferred and indeed all the examples of inkjet printed photodetectors so far reported in literature make use of this approach. In piezo-electric printheads a piezoelectric material is electrically sti-mulated causing its compression and expansion according to its resonant modes. As a result, a pressure wave propagates into the nozzle propelling a droplet out of it. Ink viscosity must be in the range of 1-30 mPas. Typical patterns deposited by inkjet printing have feature sizes of $20-50 \mu \mathrm{m}$, in the best cases, and thickness lower than $1 \mu \mathrm{m}$. Inkjet printing throughput is around $0.01-0.5 \mathrm{~m}^{2} \mathrm{~s}^{-1}$ and the registration is pretty good, with an alignment error possibly smaller than 10 $\mu \mathrm{m}$.

Inkjet printing has been employed in various fabrication processes of organic optoelectronic devices [4, 43, 44]. In this section we report on printed photodetectors realized by inkjet printing and we present examples of fully inkjet-printed devices.

The most common donor-acceptor blend employed in organic photodetectors is based on a mixture of poly(3-hexylthiophene) (P3HT) and [6]-phenyl C61 butyric acid methyl ester (PCBM), due to the extensive studies already performed on this blend in the photovoltaics field.

A first example of inkjet-printing of a P3HT:PCBM blend as photoactive layer in vertical photodetectors is shown by Lilliu et al [45]. The device structure featured a stack of glass/indium-tin-oxide (ITO) and spincoated poly(3,4-ethylenedioxythiophene) poly(styrenesulfonate) (PEDOT:PSS) as bottom electrode, a printed P3HT:PCBM blend, and evaporated calcium and silver as top electrode. They showed a responsivity of $0.32 \mathrm{~A} \mathrm{~W}^{-1}$, corresponding to $63 \%$ peak EQE (figure 1), and $D^{*}=8 \times 10^{11}$ Jones (at $5 \mathrm{~V}$ in reverse bias). In the same work, the authors replaced the spincoated PEDOT:PSS layer with an inkjet-printed one, though it showed slightly worse characteristics $\left(R=0.27 \mathrm{~A} \mathrm{~W}^{-1}\right.$, $D^{*}=3.4 \times 10^{11}$ Jones).

Similarly, by changing the top electrode with an evaporated aluminum contact, Wojciechowski et al realized a photodetector capable of working at low reverse bias 


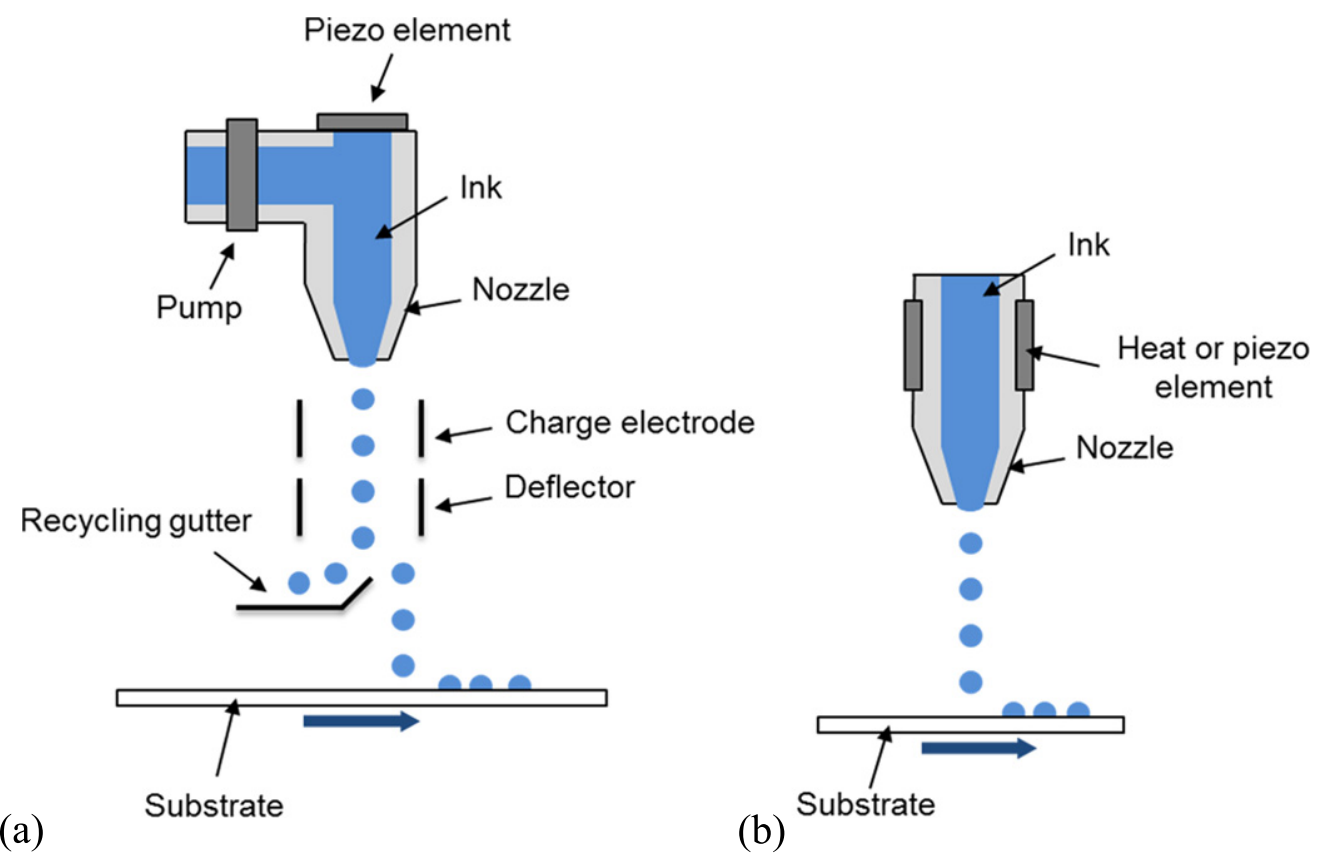

Scheme 3. Scheme of continuous (a) and DOD (b) inkjet printer nozzles.

a)

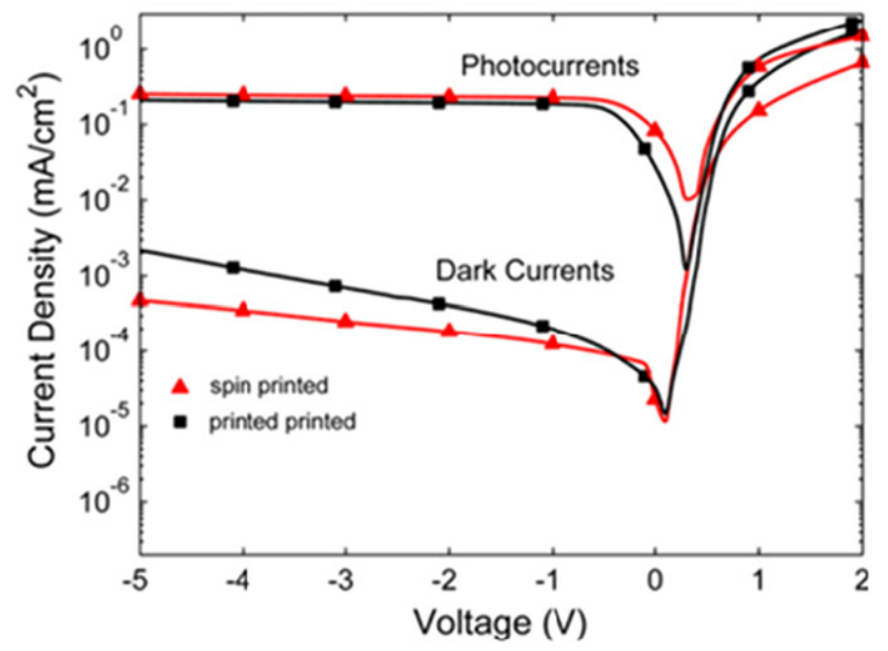

b)

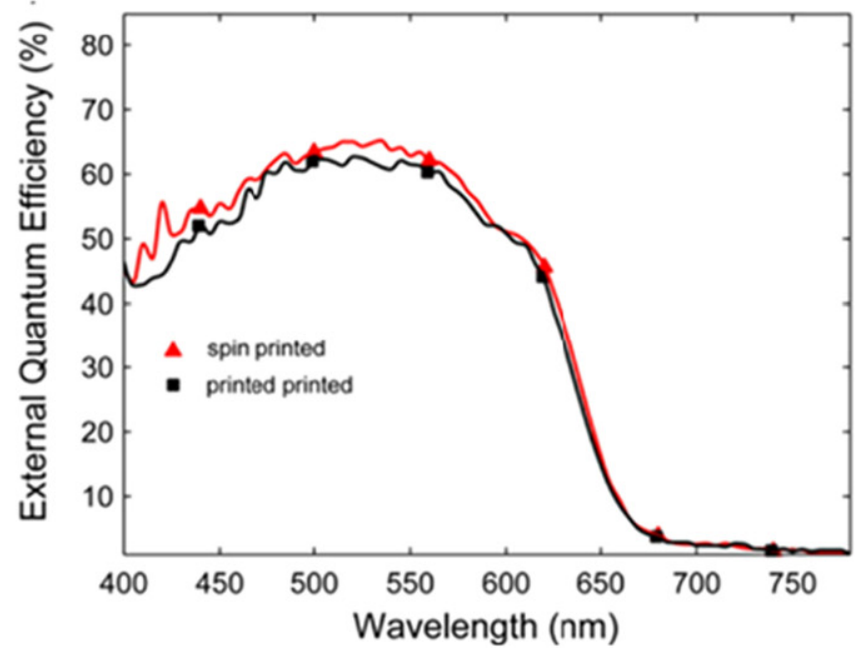

Figure 1. Comparison of the electrical characteristics and spectra sensitivity of OPDs fabricated with different layer deposition techniques by Lilliu et al [45]. (a) $I-V$ measurements of dark currents and photocurrents. (b) EQE measurements. Note: 'spin-printed' refers to the techniques used to fabricate the hole conducting and the bulk heterojunction layer, respectively. (Reprinted from [45], Copyright 2011, with permission from Elsevier.)

$\left(100 \mathrm{mV}\right.$ ) with a $R=0.25 \mathrm{~A} \mathrm{~W}^{-1}$ (at $532 \mathrm{~nm}$ ) and high $D^{*}=$ $1.4 \times 10^{13}$ Jones [35]. This device was successfully integrated into a miniaturized biosensor.

Printing methods not only allow the printing of the active materials but also of the electrodes, paving the way for the fabrication of all inkjet-printed photodetectors. The first example was published by Lavery et al [46] who succeeded in stacking silver nanoparticles (bottom electrode), a $\sim 1 \mu \mathrm{m}$ thick blend of poly $(9,9$ '-dioctylfluorinene-co-bis-N, N'-(4butylphenyl)-bis-N,N'-phenyl-1,4.phenylenediamine) (PFB) and poly(9, 9'-dioctylfluorene-co-benzothiadiazole) (F8BT) as active material and PEDOT:PSS as top electrode, depositing each layer by inkjet printing. A fluorosurfactant was added to PEDOT:PSS ink in order to allow the printing of PEDOT:PSS directly on top of the blend otherwise affected by dewetting phenomena. The photosensor was developed to work at high luminance $\left(\sim 100-400 \times 10^{3}\right.$ lux $)$ and showed an EQE peak of $5.9 \%$ at $400 \mathrm{~nm}$, dark current density of $1 \mathrm{nA} \mathrm{cm}^{-2}$ and $D^{*}=1 \times 10^{12}$ Jones (at $1 \mathrm{~V}$ reverse bias).

The low EQE in the previous example is mainly limited by the thick active layer adopted. Indeed in an all inkjet printed stack it is not trivial to achieve high quality and 
(a)

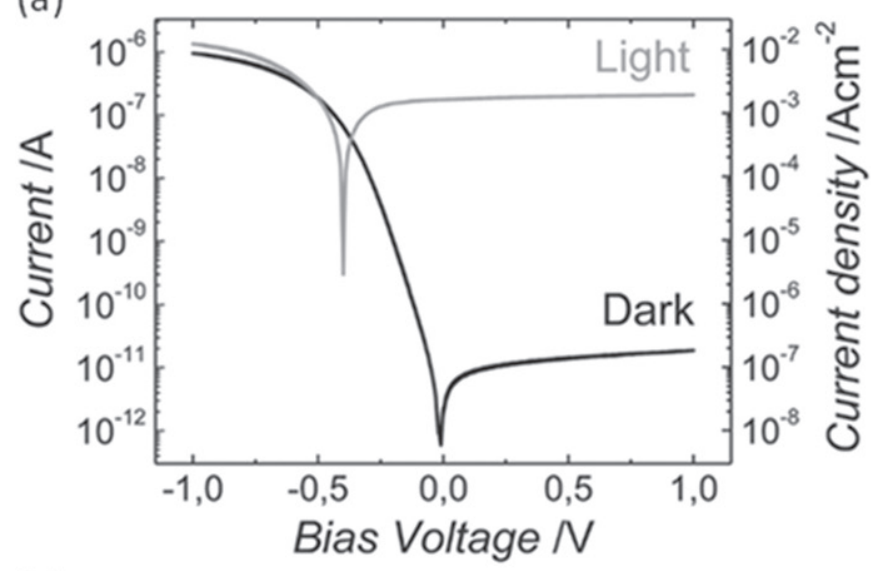

(c)

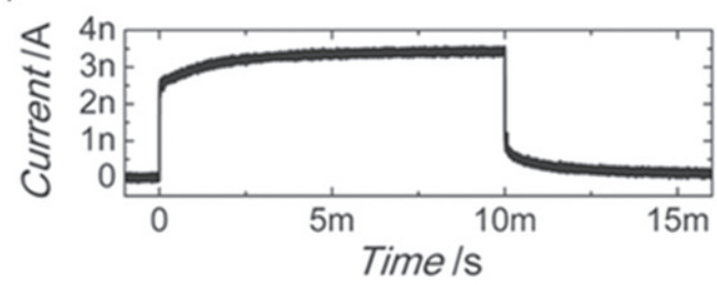

(b)

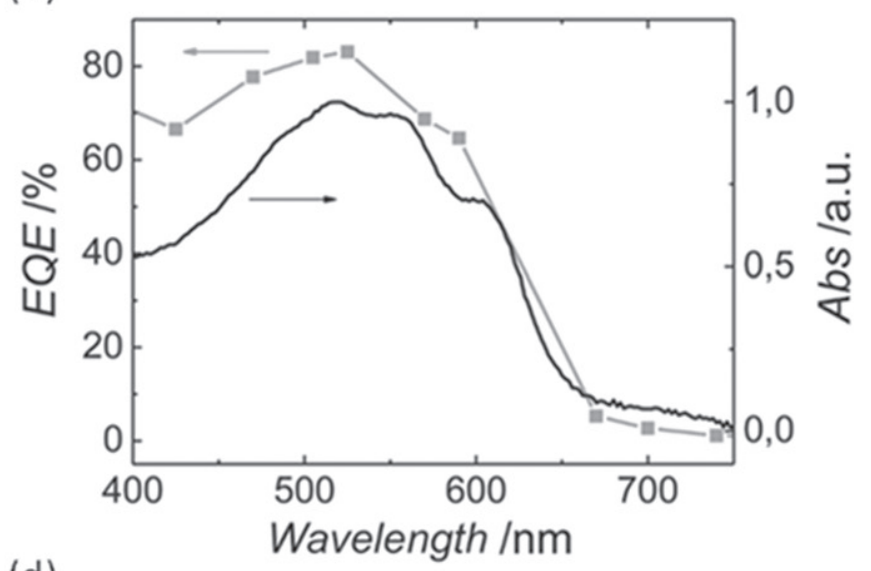

(d)

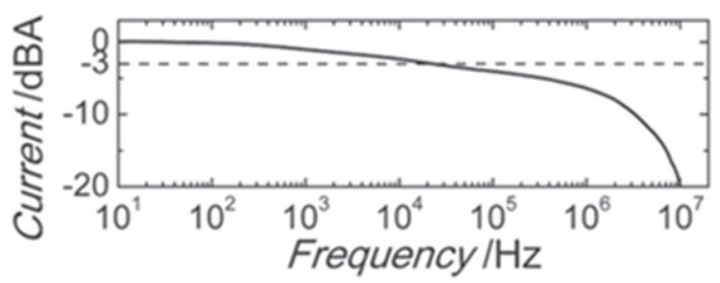

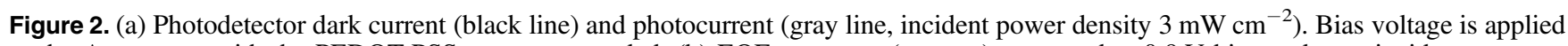
to the Ag contact with the PEDOT:PSS contact grounded. (b) EQE spectrum (squares) measured at $0.9 \mathrm{~V}$ bias under an incident power density of $10 \mathrm{~mW} \mathrm{~cm}^{-2}$ and normalized active layer absorbance (black solid line). (c) Device bandwidth measured at 570 nm at an incident power density of $0.1 \mathrm{~mW} \mathrm{~cm}^{-2}$ (1 V applied bias). (d) Bandwidth measurements. (Reproduced with permission from [36], Copyright 2013, Wiley.)

uniformity of the printed active layers to avoid short-circuits or high leakage currents between the two printed electrodes. Such limitation has been recently overcome by Azzellino et al [36] who succeeded in integrating much thinner inkjet printed P3HT:PCBM layers in a similar architecture. Thanks to the optimized film uniformity, it was demonstrated that with $\sim 120 \mathrm{~nm}$ thick films a $R=0.39 \mathrm{~A} \mathrm{~W}^{-1}$ at $525 \mathrm{~nm}$ could be achieved in an all inkjet printed detector with a reverse applied bias voltage of $0.9 \mathrm{~V}$. In order to achieve a good current rectification, it was shown that the surface modifica-tion of the bottom Ag layer was very important. Without any modification, data were hardly reproducible, with poor definition of the diode polarities, likely related to an erratic value of the printed electrode work-function. The insertion of a spincoated layer of polyethylenimine (PEIE) between the silver electrode and the photoactive blend was a crucial step to control and reduce the cathode work function. This surface treatment also affected the silver electrode wettability facilitating the controlled deposition of the active layer [36]. The level of control achieved in the printing process allowed obtaining a good fabrication yield and reproducibility. The photodetector presented a dark current density in the order of hundreds of $\mathrm{nA} \mathrm{cm}{ }^{-2}$, yielding an estimated $D^{*}=1.5 \times 10^{12}$ Jones (at $0.9 \mathrm{~V}$ reverse bias) (figure 2(a)). The authors showed that the EQE and response time were dependent on the optical power, both increasing when decreasing the impinging power. The response time ranges from $100 \mathrm{~ms}$ to $100 \mu$ s with power density increasing from $4 \mu \mathrm{W} \mathrm{cm}{ }^{-2}$ to $1 \mathrm{~mW} \mathrm{~cm}^{-2}$. As the bandwidth measurement shows (figure 2(d)), for frequency in excess of $20 \mathrm{kHz}$ a slow decrease in photocurrent occurs, while a significant fall takes place only at $1 \mathrm{MHz}$, which is an interesting result compared with the bandwidth exhibited by the other examples taken into account in this survey.

In a recent work we showed that, by properly selecting a conjugated polymer to be added to the ink formulation, it is possible to prepare printable inks of active blends comprising fullerenes and small conjugated molecules [47], opening the possibility to exploit advantages from small molecule synthesis. A blend based on a narrow bandgap conjugated molecule and fullerene was explored in the stack presented in figure 3 , where P3HT was used as an additive in the blend in order to improve the small molecule printability on flexible PEN substrates. Poly((9, 9-bis(3'-(N, N-dimethylamino)pro-pyl)-2, 7-fluorene)-(alt-2, 7-(9, 9-dioctylfluorene)) (PFN) was introduced as an interlayer between the bottom electrode and the active material, decreasing the electrode work function and enhancing its wettability. The responsivity to light was extended up to $750 \mathrm{~nm}$, owing to the photoresponse of the low bandgap molecule. In addition, by replacing the silver bottom electrode with a printed PEDOT:PSS/PFN electrode, we demonstrated the fabrication of an all-organic, semitransparent device. The ternary blend and a P3HT:PCBM one were tested in the semitransparent configuration with an EQE of $30 \%$ and $60 \%$ (at $\sim 550 \mathrm{~nm}$ ) respectively. To demonstrate the semi-transparency of the photodetector, the EQE was measured with light impinging from the front and back side (figure 3). 


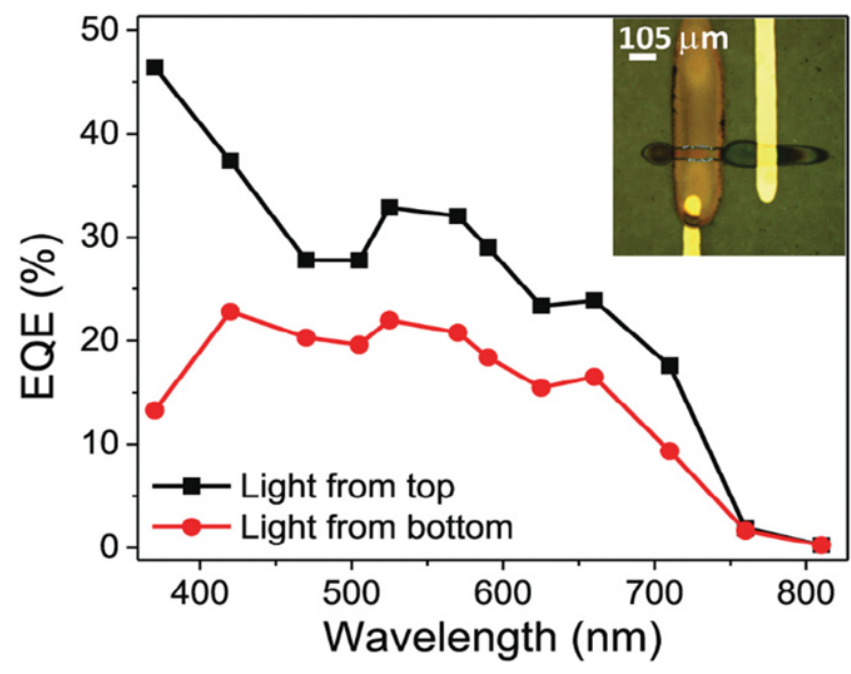

Figure 3. EQE spectra of the device containing the P3HT-small molecule blend as active material, acquired at $1 \mathrm{~V}$ bias, with light impinging either from the bottom or the top side. Inset: optical microscope image of all-organic printed photodetector [47]. (Reproduced with permission from [47], Copyright 2014, Wiley.)

Though the P3HT:PCBM blend is the most commonly employed to provide a proof of concept of the device fabrication feasibility, other photoactive materials are being explored to extend the optical window of inkjet printed photodetectors. We can find demonstration of photodetectors based on nanocrystals, J-aggregated cyanine dyes, graphene and polymeric blends [23].

HgTe-nanocrystals (NC) resulted as good candidates in photodetectors due to their response in the far and near IR and their long time stability in films. HgTe-NC dispersed in chlorobenzene form an inkjet printable solution suitable for fabrication of photodetectors. By printing this ink on interdigitated Ti/Au electrodes, Böberl et al [23] achieved a maximum $D^{*}=3.2 \times 10^{10}$ Jones, under $1 \mathrm{nW}$ of illumination at $1.4 \mu \mathrm{m}$. By changing NC size, the responsivity spectrum can be tuned, allowing the extension of the device response up to $3 \mu \mathrm{m}$. Wu et al [48] presented a thin film NC photodetector printed on paper with an office inkjet printer.

Yang et al [49] incorporated three different cyanine dyes into PEDOT:PSS to form a solution ink which was printed on top of an ITO substrate. A thin Al layer was evaporated on the PEDOT:PSS-dye blend. The red-shifted and sharp absorption spectra indicated the formation of dye's J-aggregates dispersed into the PEDOT:PSS matrix. The open-circuit voltage measurements showed a change when the light probe wavelength was around the dye absorption peak, demonstrating the possibility to detect light signals emitted from a solid-state laser. Though many examples of printed organic solar cells can be found in the previous literature [50], this work represented a first example of an inkjet printed photodetector operating on a flexible polyethylene terephthalate (PET) substrate. It is worth noting that organic solar cells can in principle be operated as photodetectors. However a good photodetecting device requires the optimization of specific figure of merits which are not as relevant or as stringent for solar cells, such as dark current minimization, detectivity, response time etc. These aspects are typically overlooked when optimizing a solar cell.

A titanium (IV) bis(ammonium lactate) dihydroxide (TBA) and graphene oxide (GO) nanosheets ionic solution was formulated and inkjet-printed on coplanar graphene-based electrodes, realizing a planar photodetector by Manga et al [51]. This ink overcomes the issues related to the poor solubility of GO in ethanol based solutions and the rapid hydrolysis of titanate by aqueous solutions, while having a suitable viscosity to allow inkjet printing. Sintering of the composite was obtained by a thermal process. The resulting device showed a peak EQE of $85 \%$ and $D^{*}=2.33 \times 10^{12}$ Jones around $325 \mathrm{~nm}$. The detector was also responsive in the visible region up to $750 \mathrm{~nm}$ but with $D^{*}$ quickly decreasing for wavelengths longer than $375 \mathrm{~nm}$, reaching values lower than $10^{12}$ Jones.

In the literature, inkjet has been explored also to fabricate all-printed detectors with inorganic active layers and carbon based electrodes. An example is given by Finn et al [52] who fabricated a planar all inkjet-printed photoconductor by using a dispersion of N-methyl pyrrolidone (NMP) exfoliated $\mathrm{MoS}_{2}$ nanosheets as photoactive semiconductor and NMP-exfoliated graphene for the interdigitated contacts. The detector, fabricated at temperatures below $70{ }^{\circ} \mathrm{C}$ on a commercial PET substrate, showed a 10 -fold current increase when exposed to $640 \mathrm{~mW} \mathrm{~cm}^{-2}$ incident illumination.

In the following we will describe a few examples where inkjet printing has been adopted for the fabrication of organic phototransistors.

A film of 6,13-bis(triisopropylsilylethynyl) (TIPS) pentacene was deposited by inkjet printing as the semiconducting material in phototransistors based on a suspended top-source and drain contacts structure [53]. Under a steady-state white light intensity between 9 and $13 \mathrm{~mW} \mathrm{~cm}{ }^{-2}$, the phototransistor produced a photocurrent over dark current ratio exceeding $10^{6}$ and $R=0.11 \mathrm{~A} \mathrm{~W}^{-1}$ at $-10 \mathrm{~V}$ of gate voltage and $1 \mathrm{~V}$ of drain-source polarization (figure 4).

Polymer phototransistors have also been realized by inkjet printing a diketopyrrolopyrrole-thiazolothiazole copo-lymer (PDPPTzBT) over a bottom gate bottom contact structure, where also a short-channel $(0.7 \mu \mathrm{m})$ was patterned by inkjetprinting [37]. To obtain such a short channel polymethylmethacrylate (PMMA) resist was spin-coated and patterned by inkjet printing pure solvent. The solvent drop dissolves the PMMA, leaving a hole or a line if the distance between subsequent droplet is reduced. Whit such a process lines of PMMA with sub-micron width can be patterned. Subsequently an adhesion layer and gold were deposited. Liftoff of the PMMA-ridge leaves a short-channel gap between gold electrodes. Such devices showed very high responsivity $\left(10^{6} \mathrm{~A} \mathrm{~W}^{-1}\right)$ under weak illumination intensity $\left(700 \mathrm{nW} \mathrm{cm}^{-2}\right.$ at $650 \mathrm{~nm}$ of wavelength, figure 5). Upon irradiation, the drain current of the phototransistor increased within $1 \mathrm{~s}$. However, when the light was turned off the drain current was not decaying to the off value but remained in a steady state for at least $10000 \mathrm{~s}$, indicating a light triggered memory effect. 


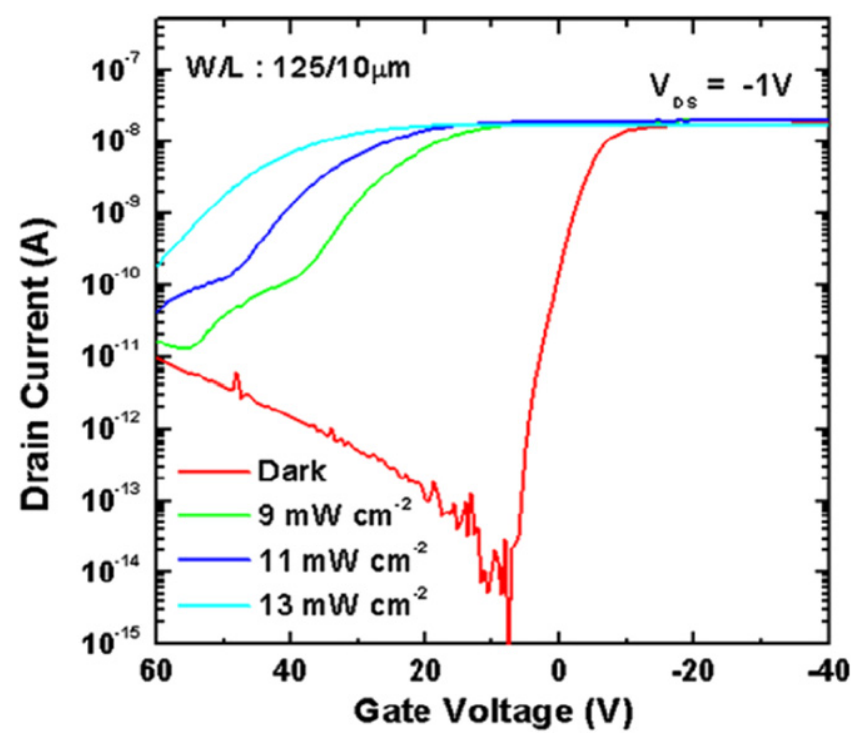

Figure 4. The transfer characteristics of a printed TIPs-pentacene phototransistor with a steady-state light illumination (at $V_{\mathrm{ds}}=-1 \mathrm{~V}$ ). The light power density was varied as $9,11,13 \mathrm{~W} \mathrm{~cm}$ ${ }^{-2}$; channel length of $10 \mu \mathrm{m}$ and a width of $125 \mu \mathrm{m}$ [53].(Reprinted from [53], Copyright 2010, with permission from Elsevier.)

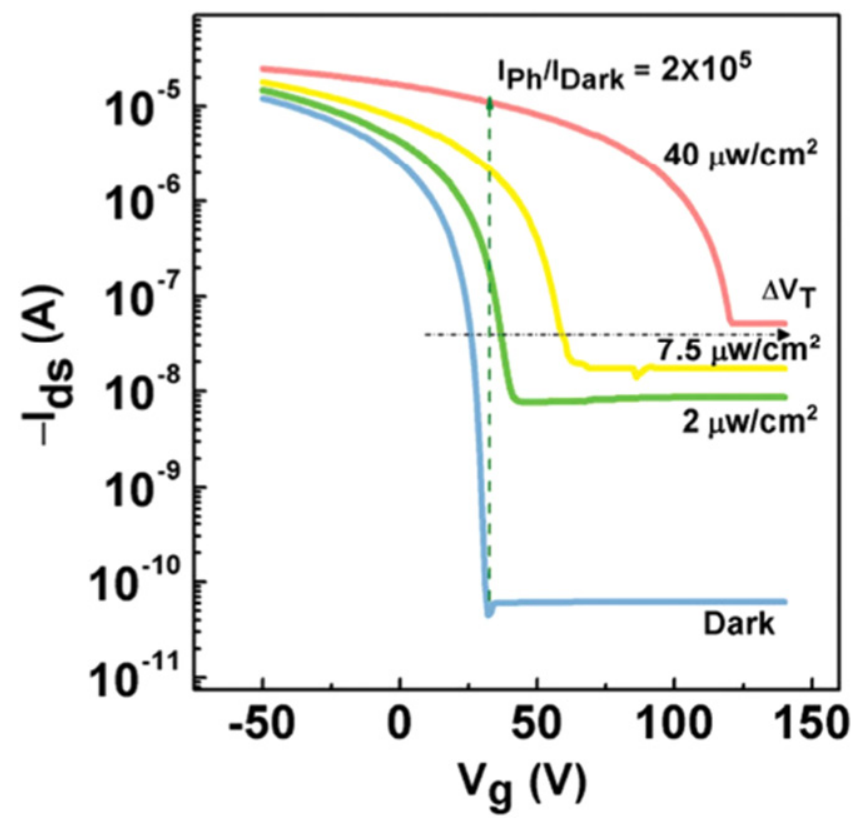

Figure 5. Transfer characteristics of the PDPPTzBT transistor under illumination compared with dark transfer curve [37]. (Reproduced with permission from [37], Copyright 2014, Wiley.)

Recently, Kim et al [38] reported all printed phototransistors where inkjet was adopted to pattern the organic semiconductor and the gate electrode. In this work, the authors showed a combination of printing methods with the intent of applying the most ideal printing technology to the desired pattern and solution formulation: besides inkjet, they adopted reverse offset printing [54], which allows for a higher lateral resolution, to pattern source and drain electrodes with a channel length of $20 \mu \mathrm{m}$ and channel width of $1000 \mu \mathrm{m}$; a simple bar-coating technique was instead adopted to achieve a uniform blank deposition of a polymeric dielectric (figure 6) [55]. The all printed phototransistors showed a responsivity of $0.11 \mathrm{~A} \mathrm{~W}^{-1}\left(V_{\mathrm{G}}=-10 \mathrm{~V}\right.$ and $\left.V_{\mathrm{D}}=1 \mathrm{~V}\right)$.

Also various inorganic light sensitive materials have been adopted to develop phototransistors with inkjet printed active layers, comprising indium doped zinc oxide (IZO) [56] and $\mathrm{MoS}_{2}$ [57], both exhibiting a photoresponse in the UV region. IZO-FET showed a current modulation of four orders of magnitude in the steady state and when exposed to a UV light pulse, and showed turn-on and turn-off times of $5 \mathrm{~ms}$ and $10 \mathrm{~ms}$ respectively.

A blend of carbon nanotubes and nanocrystal particles was employed as hybrid active layer for the fabrication of an hybrid phototransistor. Carbon nanotubes were deposited via inkjet printing, while NCs were absorbed by dipping the printed substrates in a dispersion of cadmium telluride (CdTe) [58]. While the device was exposed to light, the drain-source voltage variations were measured keeping constant the drain current at $1 \mathrm{~mA}$. The ratio between voltage variation and impinging power was found to be $20 \mathrm{mV} \mathrm{W}^{-1}$ and $15 \mathrm{mV} \mathrm{W}$ ${ }^{-1}$ for a phototransistor with and without NCs respectively.

The examples reported so far confirm the very promising use of the inkjet printing in the realization of photodetectors, allowing the achievement of good electro-optical properties comparable with other non-printing and conventional techniques, such as vacuum evaporation and spin coating. The versatility of this technique stands out clearly from the reported examples where different materials, structures and patterns were used after a proper formulation of the ink. Further developments of the technique should face the improvement in fabrication yield, resolution and throughput, to the purpose of fostering its wider exploitation into electronics industry.

\subsection{Screen printed photodetectors}

In screen printing the layer deposition is obtained by pushing a viscous ink through a mesh which is suspended over the substrate by a frame. Eventually, a stencil formed by inkblocking parts can be lean on the mesh. A soft blade called squeegee is moved across the screen forcing the ink ejection through the mesh openings and the substrate wetting during the squeegee stroke (scheme 4). Typically, screen printing allows lateral feature sizes in the range of $20-100 \mu \mathrm{m}$ and a wet layer thickness of 30-100 $\mu \mathrm{m}$. However, the dried layer thickness and width will be only a fraction of these values depending on the initial solid content of the ink and the substrate wettability properties. Screen printing has the advantage of being a technique compatible with roll-to-roll processes. It can be successfully exploited if the formulated ink is viscous and not volatile. Examples of screen printed photodetectors reported so far are still limited to $\mathrm{x}$-ray radiography. In the field of direct detection, it is particularly important to exploit thick films because the absorption coefficient in X-rays spectral region is lower than in the visible one. The simplicity of screen printing technique could enable 

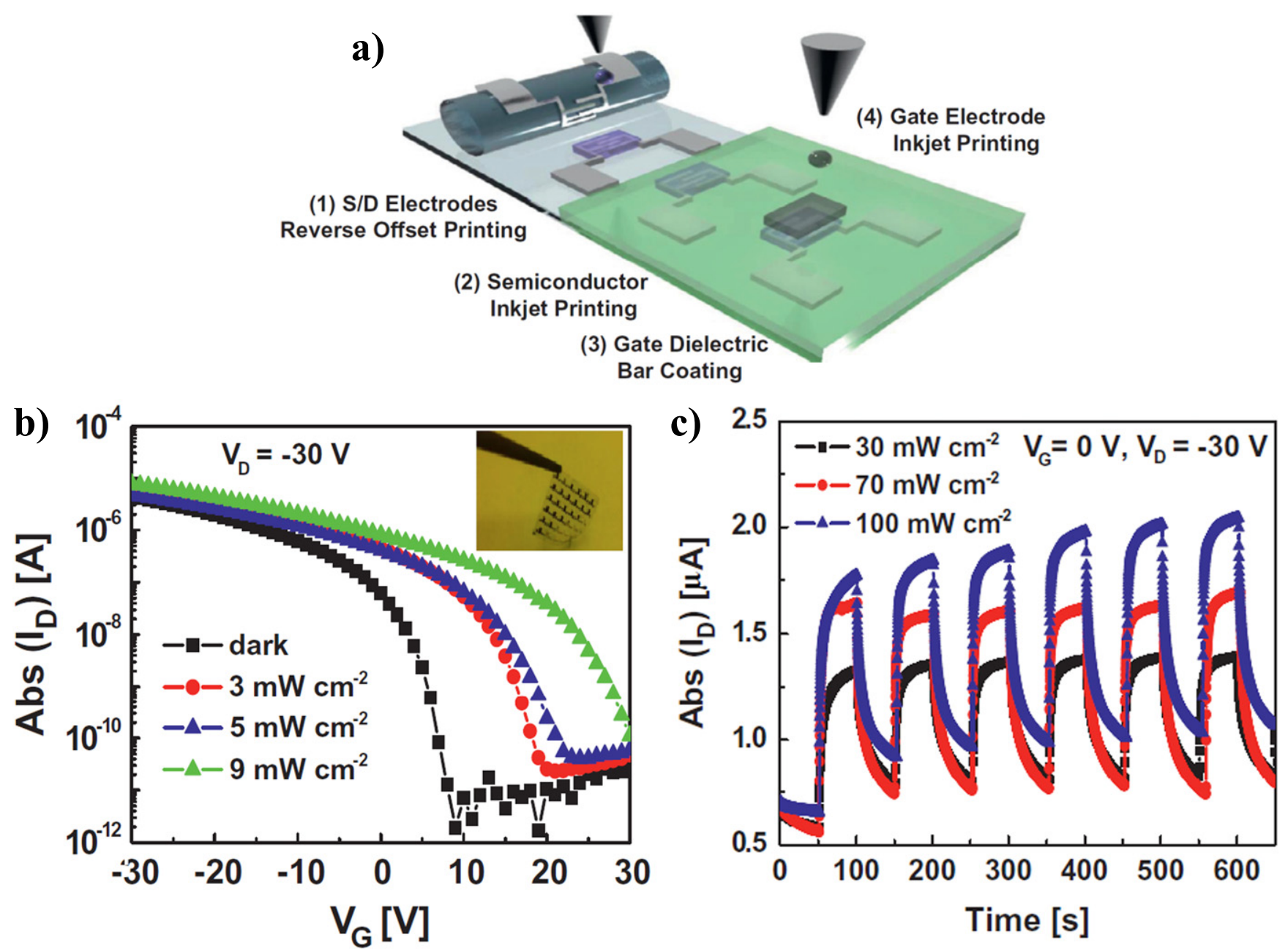

Figure 6. (a) Schematic of the combination of printing methods employed for the organic phototransistor fabrication; (b) transfer

characteristics in the dark and under illumination at different light intensity at $V_{\mathrm{D}}=30 \mathrm{~V}$ (inset: photograph of devices on flexible substrate); (c) time-resolved photo-response characteristics upon different irradiation $\left(V_{\mathrm{G}}=0 \mathrm{~V}\right.$ and $\left.V_{\mathrm{D}}=30 \mathrm{~V}\right)$ [38]. (Reprinted from [38], Copyright 2014, with permission from Elsevier.)

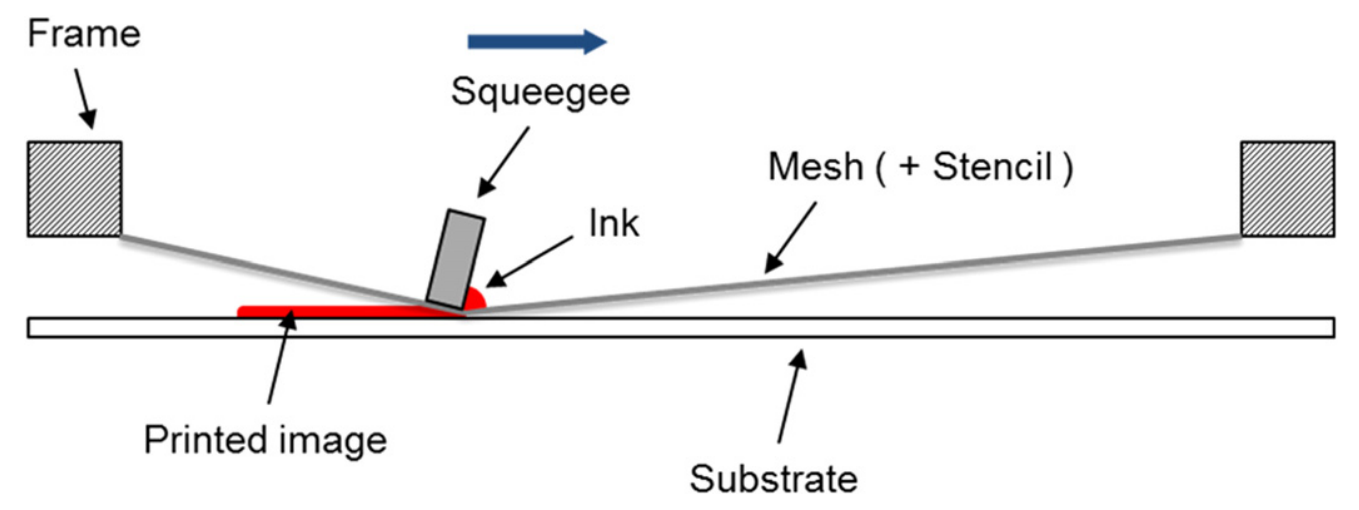

Scheme 4. Scheme of a screen printer setup.

applications in other larger scale printing technology. The throughput is $2-3 \mathrm{~m}^{2} \mathrm{~s}^{-1}$ for the screen-to-screen, while in a roll-to-roll fabrication tens of $\mathrm{m}^{2} \mathrm{~s}^{-1}$ can be obtained.

Among the technique drawbacks, it can be noted that it produces more waste of material with respect to a directwriting technique such as inkjet. The high viscosity required for screen printing inks (1000-10000 mPas) often forces the use of binders employed as additives in the formulation. Layer-to-layer registration is a major source of error (alignment error $25 \mu \mathrm{m})$.

In the following we report on the fabrication of photodetectors via screen printing methods, which mostly relate to 
a)

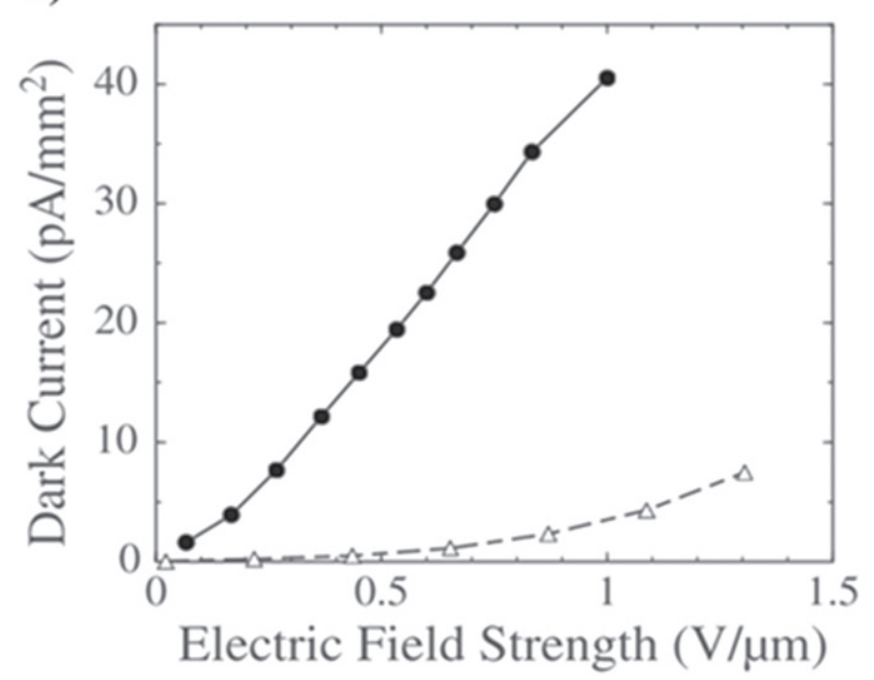

b)

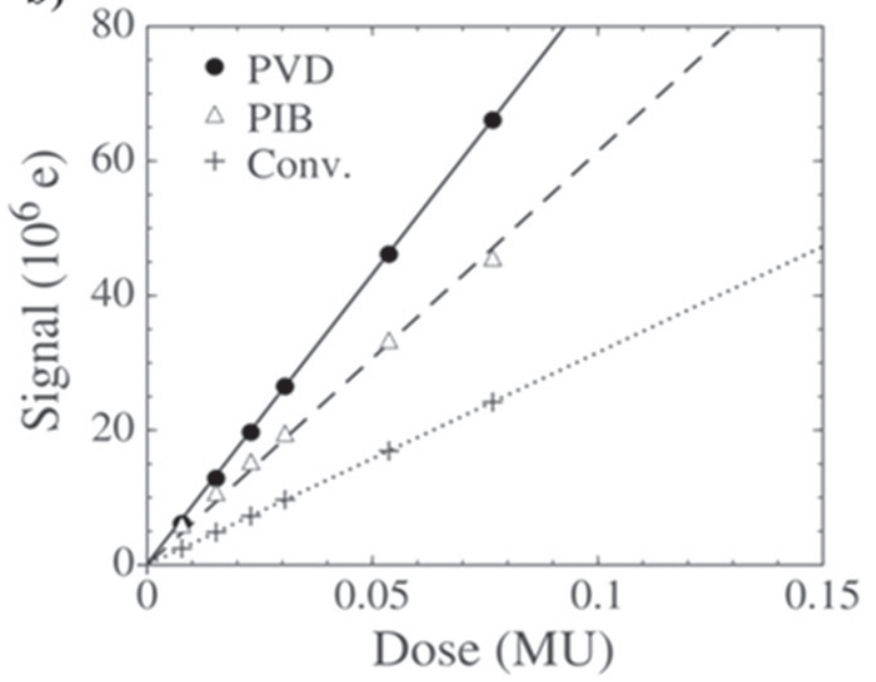

Figure 7. (a) Measurements from the $\mathrm{HgI}_{2}$ PIB prototype (triangles) and the PVD prototype (circles) of x-ray signal dark current response (the results are plotted as function of the electric field strength, calculated from the magnitude of the bias voltage applied across the photoconductor divided by the thickness of the $\mathrm{HgI}_{2}$ layer). (b) Signal detected on the $\mathrm{HgI} 2$ based pixels in the PVD and PIB prototypes. For comparison, the signal from the conventional active matrix flat panel imager designed for megaelectronvolt radiation using a Lanex FAST B screen is presented. (Reproduced with permission from [65], Copyright 2010, Am. Assoc. Phys. Med.)

the deposition of thick active layers of inorganic compounds. We will show photoconductors where a particle-in-binder was employed to enable printing.

Sebastian et al [59] reported the fabrication of planar photodetectors based on a blend of $\mathrm{CdS}$ and CdSe nanoparticles, forming a ternary composite $(\mathrm{Zn}, \mathrm{Cd}) \mathrm{S}$, by screen printing the semiconductor paste through a polyester screen of $120 \mathrm{~T}$ mesh (120 threads per centimeter) onto a glass or $\mathrm{Ti}$ substrate. Propylene glycol was used as a binder. Different concentration ratios of $\mathrm{CdCl}_{2}$ versus $\mathrm{ZnS}$ and $\mathrm{ZnCl}_{2}$ versus $\mathrm{CdS}$ were tested. It is shown that such ratio influence the electro-optical characteristics of the film. The screen printed films were sintered after their deposition for 1 hour, and their structure, composition and optoelectronic characteristics were studied by changing sintering temperature and sintering atmosphere. The best performing ( $\mathrm{Zn}, \mathrm{Cd}) \mathrm{S}$ ternary composite was the one formed by a $\mathrm{ZnCl}_{2}$ to $\mathrm{CdS}$ ratio of 0.2 , sintered at $400{ }^{\circ} \mathrm{C}$, yielding a $D^{*}$ of $1.8 \times 10^{11}$ Jones and a dark current density of $1.4 \mathrm{nA} \mathrm{cm}^{-2}[60,61]$.

Within $\mathrm{CdS}$ detectors the largest responsivity of $4.25 \mathrm{~mA}$ $\mathrm{W}^{-1}$ was obtained for a flux $\left(\mathrm{CdCl}_{2}\right)$ to semi-conductor $(\mathrm{CdS})$ ratio $\left(F / S\right.$ ratio) of 0.1 and sintering at $400{ }^{\circ} \mathrm{C}$ in air, with a dark current density of $100 \mathrm{nA} \mathrm{cm}{ }^{-2}$. For such devices a detectivity of $2.4 \times 10^{10}$ Jones was achieved [59]. Even better performances were obtained for $\mathrm{CdSe}$ devices by mixing $\mathrm{Cd}$ and Se powder at weight ratio of $2 / 3$ and sintering the screen printed layer at $500{ }^{\circ} \mathrm{C}$. Following these processing conditions the authors measured a dark current density of $20 \mathrm{nA} \mathrm{cm}^{-2}$ and $D^{*}=1.9 \times 10^{10}$ Jones, while the highest photocurrent density was obtained for a sintering temperature of $400{ }^{\circ} \mathrm{C}$ [59].

Screen printing was also employed to deposit thick films of mercuric iodide $\left(\mathrm{HgI}_{2}\right)$ mixed with polyvinyl-butyral
(PVB) as binder and a volatile solvent. $\mathrm{HgI}_{2}$ layer was exploited as a photoconductor for x-ray detection [62-64].

The comparison between screen printed $\mathrm{HgI}_{2}$ (PIB), physical vapor deposited $\mathrm{HgI}_{2}$ (PVD) and conventional amorphous selenium (a-Se) detectors was reported by Zhao et al [65] $\mathrm{HgI}_{2}$ was deposited on amorphous silicon thin film transistor (TFT) arrays and covered with a palladium electrode to form a vertical structure. For efficient charge collection both $\mathrm{HgI}_{2}$ photoconductors can be operated with an applied electric field of $1 \mathrm{~V} \mu \mathrm{m}^{-1}, 10$ times lower with respect to the one needed to drive the a-Se detector (figure 8). For a 6 $\mathrm{MeV}$ light beam the measured sensitivity were 14.8, 9.9 and $5.4 \mathrm{pC} \mathrm{mR}^{-1} \mathrm{~cm}^{-2}$ for PVD, PIB and a-Se respec-tively. Among the $\mathrm{HgI}_{2}$ sensors, the PIB showed a dark current density of $400 \mathrm{pA} \mathrm{cm}^{-2}$ (figure 7(a)), an order of magnitude lower than the PVD one. DQE of around $1.25 \%$ was measured on PIB and PVD prototypes, which was higher than the a-Se one and independent on light power intensity, indicating inputquantum-limited behavior, in accordance with simulation results (figure $7(\mathrm{~b})$ ). On the contrary, $\mathrm{HgI}_{2}$ photodetectors image lag is worse than for a-Se detectors being 20\%, $15 \%$ and $5 \%$ for PIB, PVD, and a-Se respectively. Arrays addressed by amorphous silicon TFT (ND10 and MD88 from dpiX, USA) were tested at $72 \mathrm{kVp}$. A sensitivity of 3.3 and $1.1 \mathrm{pC}$ $\mathrm{mR}^{-1} \mathrm{~cm}^{-2}$ was measured for PVD and PIB respectively, and DQE around 0.6 was recorded for a PVD array [62].

Kim et al [66] simulated the DQE of CdZnTe, $\mathrm{HgI}_{2}, \mathrm{PbO}$ and a-Se photoconductors finding $\mathrm{HgI}_{2}$ as the one which shows the highest sensitivity at $100 \mu \mathrm{m}$ film thickness and the highest DQE for low X-ray doses. Fabricating the detector in a vertical structure on top of an a:Si TFT array, after testing various screen printed films obtained under different conditions, the authors selected the $\mathrm{HgI}_{2}$ paste composition (PVB 

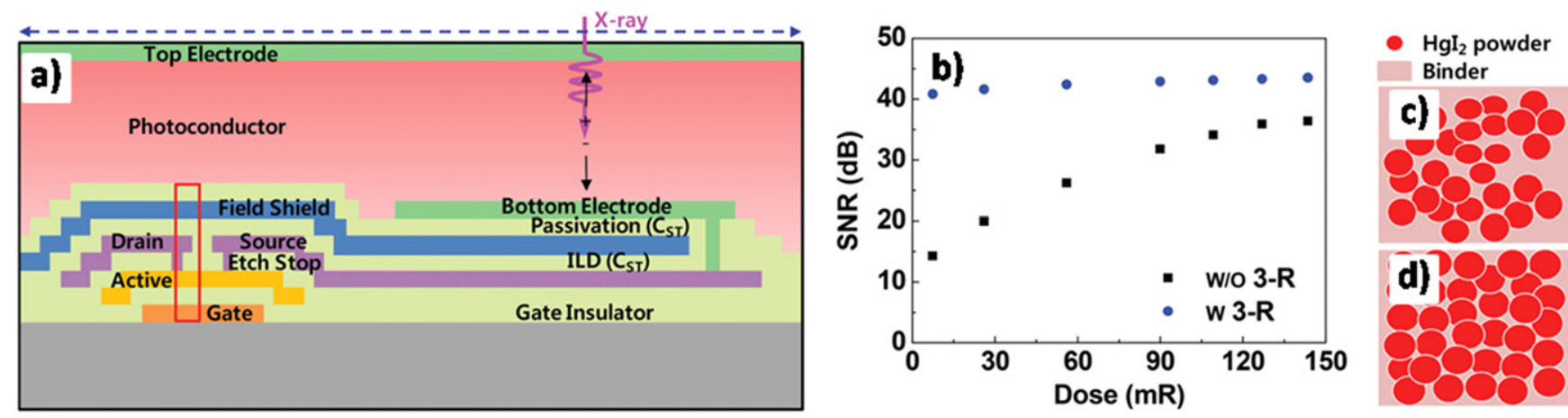

Figure 8. (a) A schematic cross-sectional image of a pixel showing the photoconductor diode and the thin film transistor (TFT) structure; ILD represents the interlayer dielectric, $C_{\mathrm{ST}}$ is the storage capacitor. (b) SNR of $\mathrm{HgI}_{2} / \mathrm{a}$-Si TFT-combined x-ray flat panel image sensor. The plots show the different SNR as a function of x-ray dose, obtained following two different preparations for the screen printed photoconductor: with (w) (c) and without (w/o) three-roll mill (d). (Reproduced with permission from [67], Copyright 2015, Wiley.)
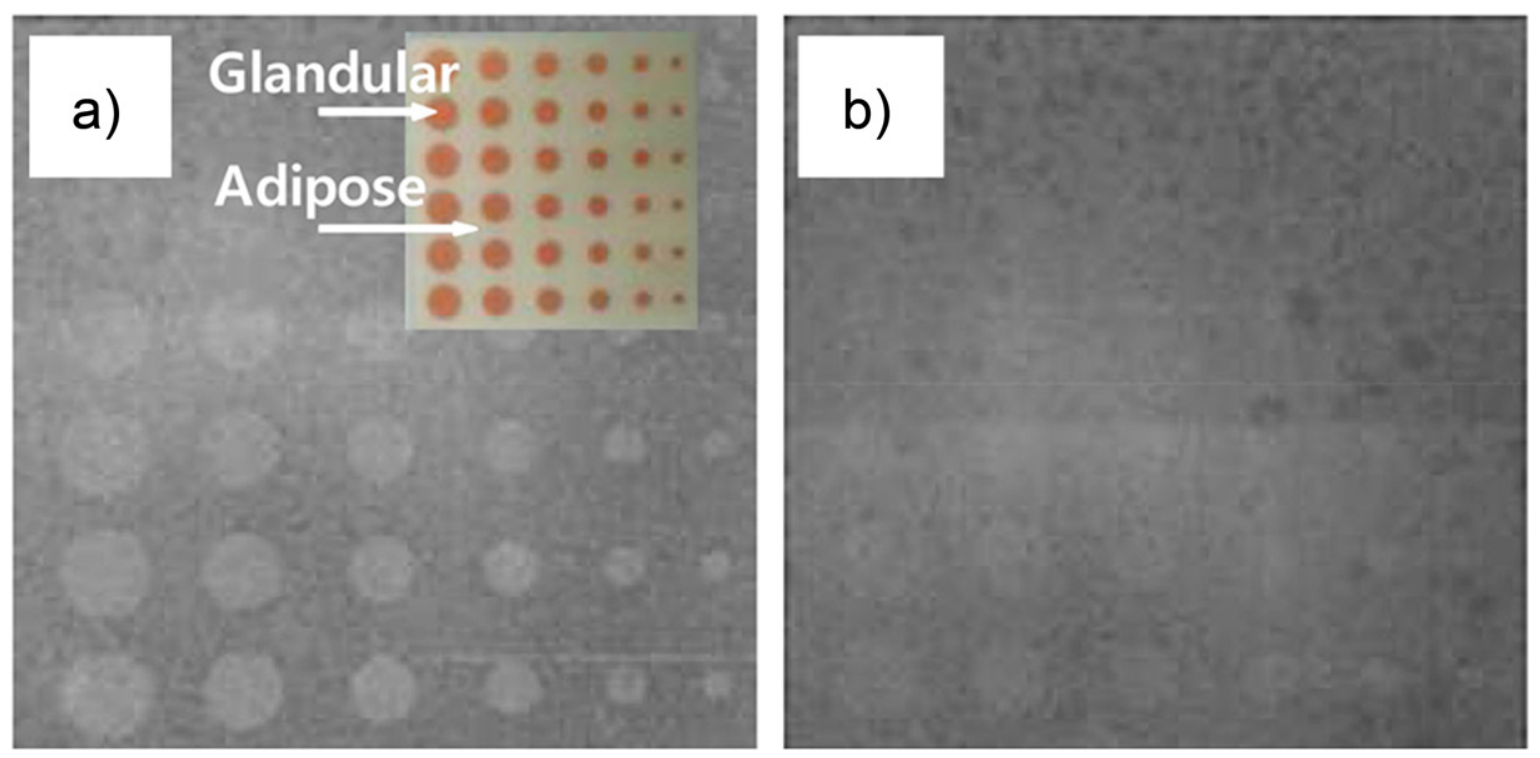

Figure 9. Comparison between $\mathrm{x}$-ray images taken either by (a) the $\mathrm{HgI}_{2} / \mathrm{a}-\mathrm{HfInZnO-based} \mathrm{flat} \mathrm{panel} \mathrm{x-ray} \mathrm{image} \mathrm{sensors} \mathrm{or} \mathrm{by} \mathrm{(b)} \mathrm{the} \mathrm{a-Se/}$ a-Si-based flat panel x-ray image sensors. The sample imaged was an artificial human textile (phantom), which is a substitute designed to match the x-ray beam attenuation in a human breast. The photograph in the inset of figure (a) shows the differences in tissue structure which the imager is able to distinguish. (Reproduced with permission from [67], Copyright 2015, Wiley.)

as binder at $10 \mathrm{wt} \%$ and butyl cellosolve as solvent) and the screen printed ruthenium as top electrode material. A sensitivity of $750 \mathrm{pC} \mathrm{mR} \mathrm{mR}^{-1} \mathrm{~cm}^{-2}$ at $30 \mathrm{kV}$ radiation energy, dark current below $3 \mathrm{nA} \mathrm{cm}{ }^{-2}$ at $1.5 \mathrm{~V} \mathrm{\mu m}^{-1}$ and $4 \%$ image lag were achieved.

A high performance direct-conversion flat panel $\mathrm{x}$-ray image pixel sensor was demonstrated by screen printing $\mathrm{HgI}_{2}$ as photoconductor [67]. The $\mathrm{X}$-ray image sensor is made of a pixels array, where each pixel combines a vertical photoconductor where $\mathrm{HgI}_{2}$ is the photosentisitive material and a TFT switch based on a-HfInZnO TFT. A schematic vertical cross-section of the pixel is presented in figure 8 , along with the SNR of the sensor as a function of x-ray dose and photo-

conductor preparation. In figure 9 the improvement in image sensing of this imager $\left(\mathrm{HgI}_{2} / \mathrm{a}\right.$-HfInZnO-TFT) is shown in comparison to the one obtained with a more conventional $\mathrm{HgI}_{2}$ /a-Si TFT-combined x-ray flat panel image sensor.

\subsection{Other non-contact printing techniques}

Though spraying is a coating technique, rather than a printing method, it is commonly discussed in the context of printed electronics [2, 6], since it can enable high throughput, low manufacturing costs and the use of a wide range of substrate materials and geometries. The main parameter to be controlled in spray coating are the colloidal nature of the particles, the liquid solvent and the gas medium, the latter enabling the dispersion of the colloidal particles in air and their delivery to the substrate [6]. So far the fabrication of organic devices by means of spray technologies has mostly included air-assisted and ultrasonic spray technology [68]. Air atomizers, or pressure nozzles, typically consist of a spray gun connected to a pneumatic controller which causes the solution atomization, by pulling the liquid through the nozzle orifice (scheme 5). This causes the sheared liquid to spread in 


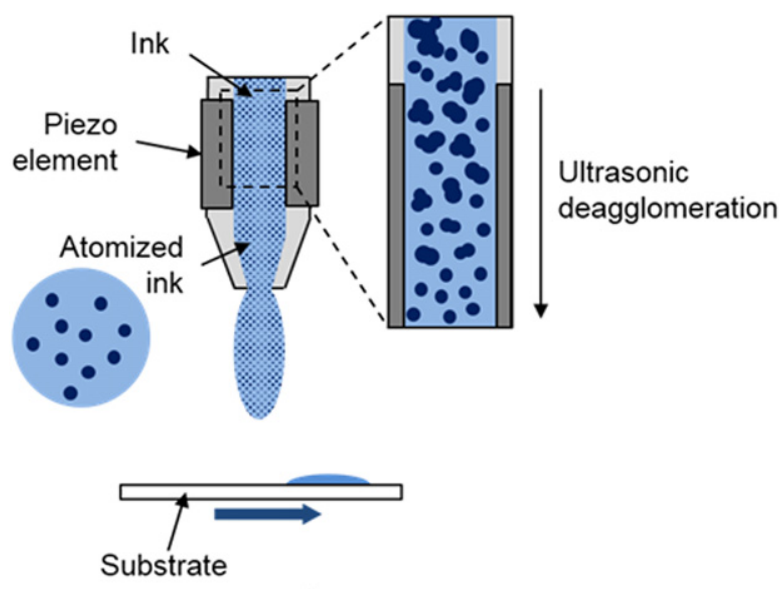

a)

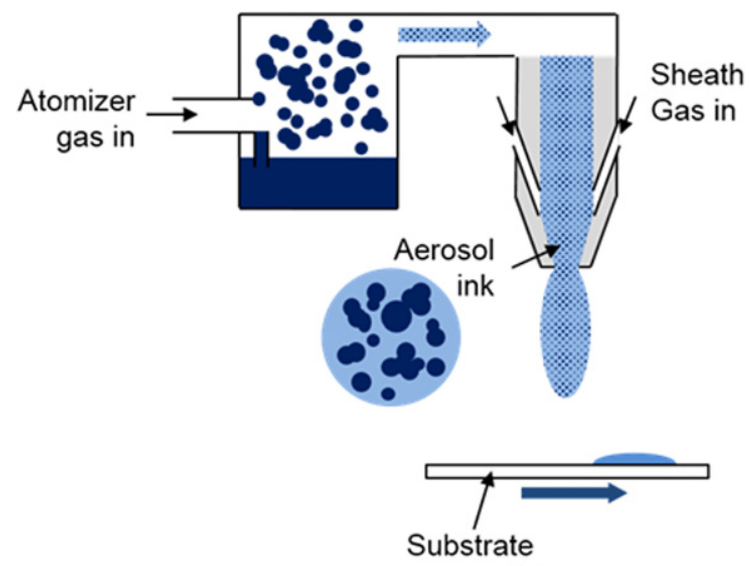

b)

Scheme 5. (a) Cross section of an ultrasonically atomized droplet. (b) Air atomization, cross section of an air spray droplet.

all directions with a wide drop size distribution, resulting in non-uniform pattern deposition and increased probability of nozzle clogging.

Ultrasonic spray coating uses ultrasonic nozzles where a piezoelectric transducer creates mechanical vibrations, so that shock waves are transferred to the material to be sprayed. Therefore by controlling the resonant vibrations of the piezoelectric nozzle, well defined atomized droplets can detach and more uniform and repeatable coatings can be achieved. Also, ultrasonic spray coating enables the release of soft, low velocity atomized spray, while a limited maintenance of the setup is necessary due to a lower probability of nozzle clogging due to aggregates formation and precipitation at the nozzle orifice.

The droplets can reach the substrates already dry. In such case there will be no facile rearrangement of the particles onto the substrates and a non-uniform layer is likely formed. On the opposite, if the drops are too wet they can easily dissolve the bottom layer, especially if it is composed of an organic material. Surface wettability and drop surface tension are therefore important factors to be controlled also in spray techniques. Drop size is governed by the surface tension and the density of the liquid being atomized. Control parameters for the spray deposition are the flow rate, the atomizing gas (usually inert $\mathrm{N}_{2}$ ), the pressure, nozzle to sample distance, substrate temperature and substrate motion speed. The control of the drying process can occur by raising the substrate temperature during deposition or also by flowing more inert gas, as this can be crucial to reduce coffee ring effects inducing unevenly patterned surfaces. In spray coating techniques it is critically important to control the dynamic of the solution atomization so that the film drying at the surface can provide a smooth morphology: this is one of the main issues to be solved case by case depending on the nature of the ink formulation. Though spray coating technique is limited by its lower line shape accuracy and resolution with respect to other printing methods, it has proven to be successfully employed for photodetectors fabrication and can possibly enable a high throughput in a scaled up production.
P3HT:PCBM based photodetectors where all the organic layers have been spray coated have been reported by Tedde $e t$ al [40]. They showed that in spite of the higher surface roughness characterizing the spray coated films, the device performance improved with respect to the films obtained by spin coating and doctor blade techniques. Spray coated detectors achieved a peak $\mathrm{EQE}=71 \%$ at $550 \mathrm{~nm}$ (corresponding to $R=0.36 \mathrm{~A} \mathrm{~W}^{-1}$ ). The detectivity of the devices reached $8.8 \times 10^{11}$ Jones $(-5 \mathrm{~V}, 532 \mathrm{~nm})$. For the same device a $3 \mathrm{~dB}$ cut-off frequency was measured at $100 \mathrm{kHz}$.

A non-contact technique such as spraying enables the development of hybrid technology, where a solution-processed photoactive layer is coupled with conventional silicon based CMOS technology. Hybrid CMOS-imagers were demonstrated by Baierl et al by spraying a photoactive P3HT:PCBM layer onto a silicon based CMOS-pixel array, demonstrating simple imaging achieved with $30 \times 30$ hybrid pixels resolution as shown in figure 10 [69]. This strategy enabled to achieve a geometrical fill factor up to $100 \%$, with an EQE of $40 \%$ (at $600 \mathrm{~nm}$, bias $=-2.5 \mathrm{~V}$ ), and high dynamic range $(60 \mathrm{~dB})$. The $\mathrm{BW}$ of such devices was shown to be $15 \mathrm{kHz}$, allowing a sufficient frame rate for real-time imaging applications.

Spray coating has been employed also for the deposition of transparent indium free electrodes. The ink formulation to that purpose can be based on PEDOT:PSS [70], PEDOT: PSS/ graphene [71] and carbon nanotubes [68, 72]. This enabled the fabrication of fully spray coated OPDs on the flexible PET substrate, as shown by Falco et al [73, 74]. The vertical photodiode contained a sprayed coated layer of P3HT:PCBM blend as active material. The bottom electrode was composed of carbon nanotubes spray coated on the PET substrate, topped with a layer of sprayed PEDOT:PSS, while the top electrode was again sprayed PEDOT:PSS. The bottom PEDOT:PSS was employed to reduce the substrate rough-ness, avoiding short circuits between the bottom and top electrodes caused by carbon nanotubes. As mentioned above, the nature of the sprayed solution has to be colloidal, and to achieve that nanotubes have to be mixed with a proper dis-persant reactant. In their work the authors [73] found an 
a

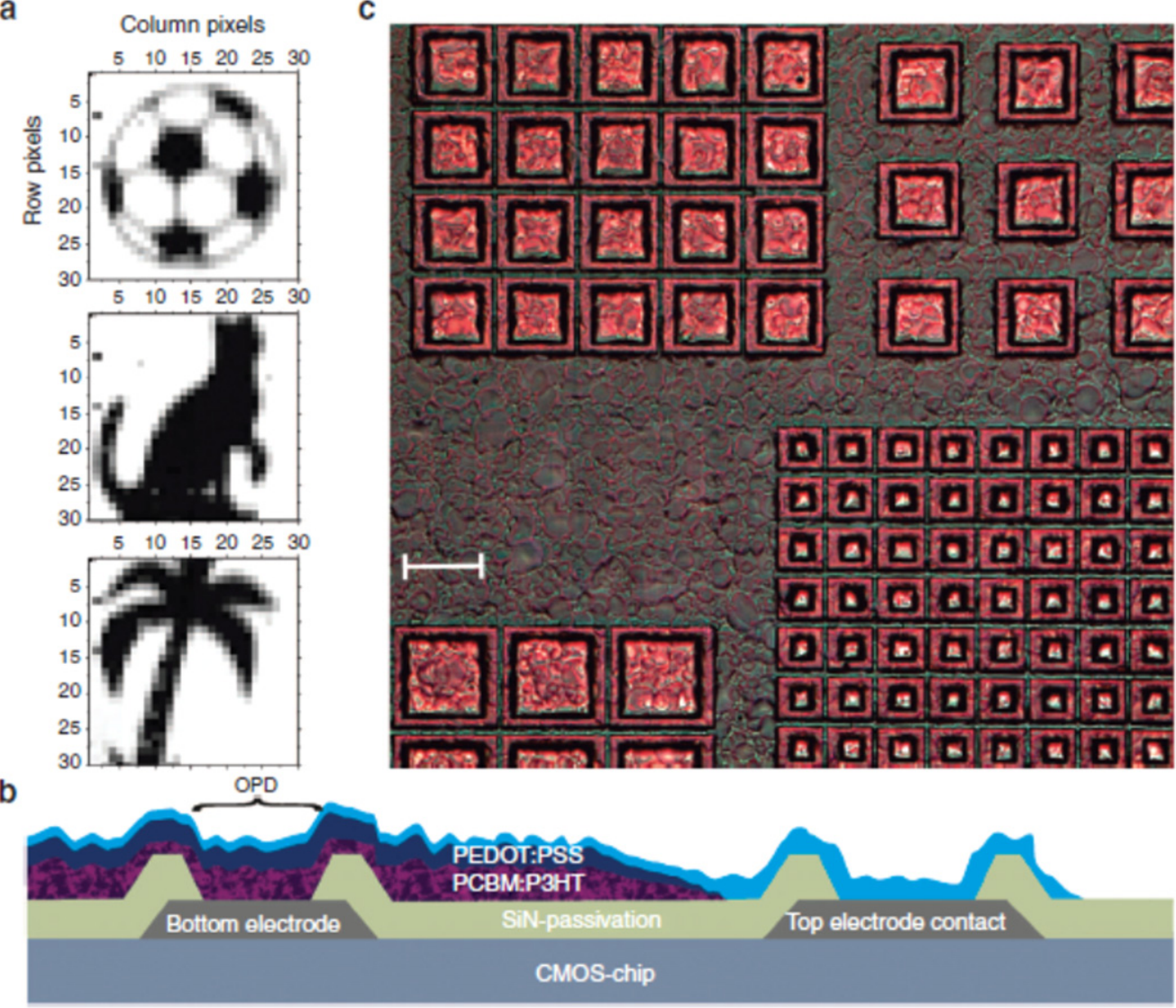

Figure 10. (a) Monochrome images acquired by the $30 \times 30$ pixel array showed in figure (c). (b) Scheme of the CMOS-organic polymer integrated device. (c) Top view of the pixels array constituting the hybrid imager. The scale bar is $50 \mu \mathrm{m}$ wide [69]. (Reproduced with permission from [69], Copyright 2012, Nature.)

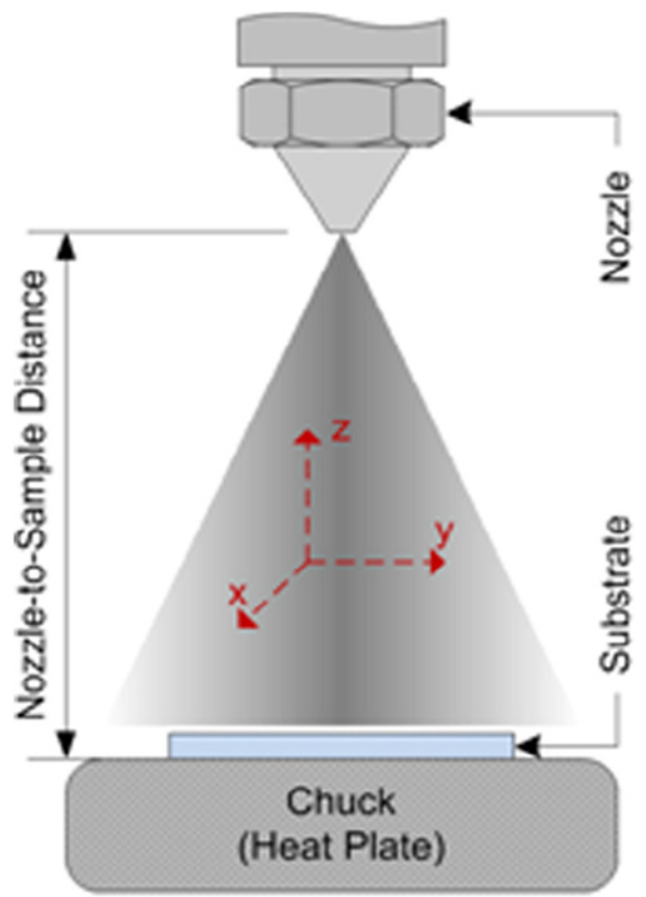

Figure 11. Schematic representation of the spray coating set-up used for the deposition of the carbon nanotubes based electrodes on to flexible substrate [73]. (Reprinted with permission from [73]. Copyright 2014, American Chemical Society.) optimum in film deposition when using carboxymethyl cellulose as the dispersant agent. Figures 11 and 12 report the schematic of the air-pressure nozzle setup used in the work, the OPD $I / V$ curves and the EQE spectra $(\mathrm{EQE}=65 \%)$.

An alternative to carbon nanotubes electrodes is the deposition of $\mathrm{ZnO}$ electrodes. UV photodetectors containing a spray coated $\mathrm{ZnO}$ films have been shown by various groups $[41,75]$. In these examples $\mathrm{ZnO}$ was employed both as electrode and as active material upon doping. In this last example [75], by doping the $\mathrm{ZnO}$ with nitrogen, a responsivity of $530 \mathrm{~A} \mathrm{~W}^{-1}(5 \mathrm{~V}, 365 \mathrm{~nm})$ could be reached.

Binda et al [42] integrated an organic photodiode onto a plastic optical fiber, thus realizing a fiber-receiver integrated system, by a spray assisted layer by layer deposition of the photodiode directly on the polished fiber surface, thus achieving an inherent alignment and solving at the roots any issue related to optical coupling. To this purpose spray coating could offer its intrinsic advantage to enable the material deposition on essentially any surface geometry, something not achievable with conventional spin-coating or epitaxial techniques and not so easily achieved via jet-printing techniques either (figure 13). The fabrication of the photodiode directly on the fiber allowed an efficient transduction of the signal with no interference from intermediate crossing medium, as for example air, favoring the best geometrical 

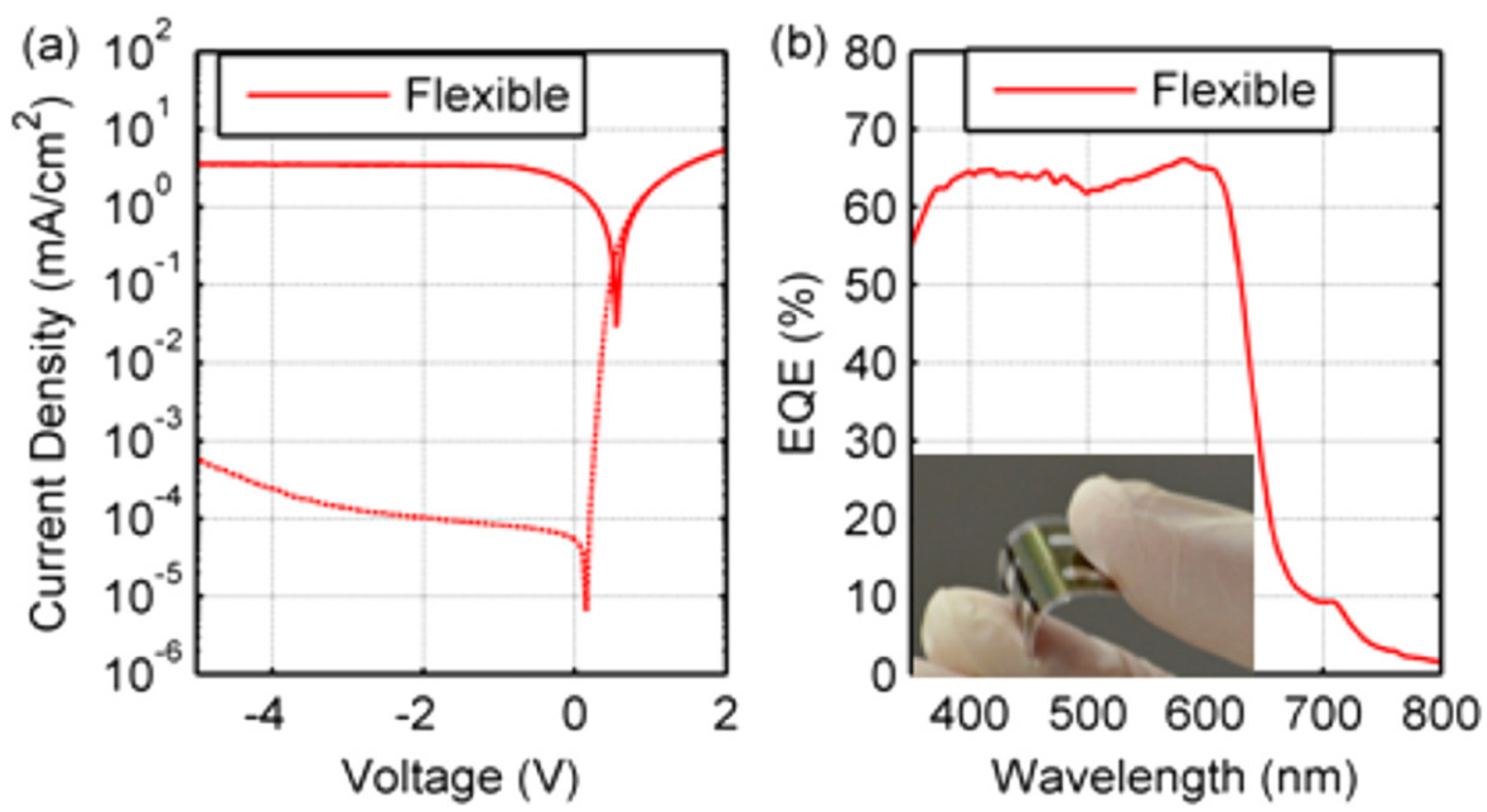

Figure 12. (a) Dark (dashed lines) and illuminated (solid lines) $J V$ characteristics of OPDs obtained with the spray coating of PEDOT:PSS and spin coating of the blend on a PET substrate. (b) EQE measured on a flexible diode (Inset). (Reprinted with permission from [73]. Copyright 2014, American Chemical Society.)

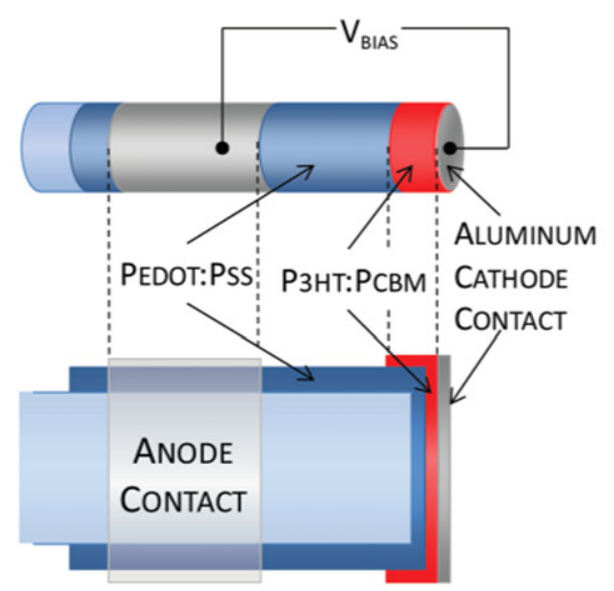

(a)

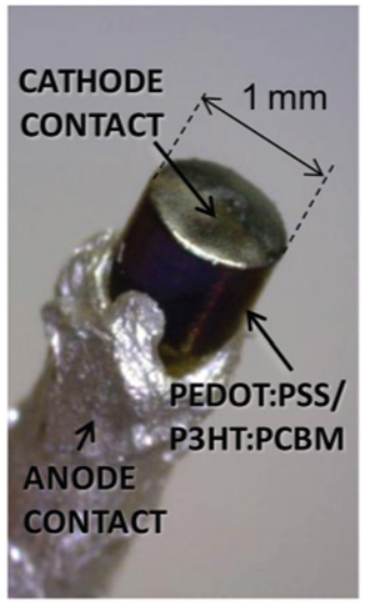

(b)

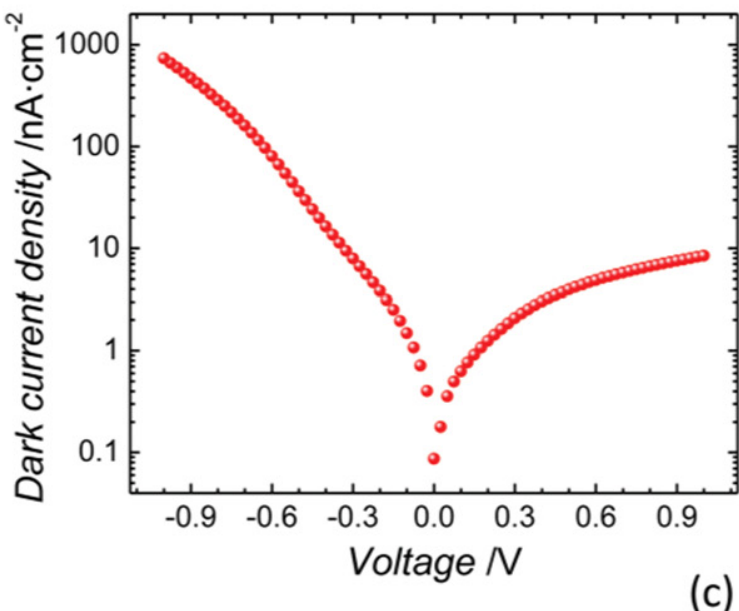

Figure 13. (a) Sketch of the integrated plastic optical fibers /OPD system and schematic of its cross section. (b) Photograph of the integrated POF/OPD prototype. (c) OPD dark current [42]. (Reproduced with permission from [42], Copyright 2014, Wiley.)

optical coupling $(\mathrm{EQE}=2 \%$ at $660 \mathrm{~nm}$ for up to $100 \mu \mathrm{W} \mathrm{cm}{ }^{-2} ; 3 \mathrm{~dB}$ cut-off frequency at $15 \mathrm{kHz}$ ).

Examples of spray-coated inorganic photoactive layers can be found in the literature as well. Zhao et al [39] demonstrated the achievement of fast (up to $1 \mathrm{MHz}$ of bandwidth at $635 \mathrm{~nm}, 800 \mathrm{~mW} \mathrm{~cm}^{-2}$, at $5 \mathrm{~V}$ bias) photodetectors deposited via spray coating of aqueous HgTe-QDs (figure 14). They also showed the long term air stability of these QDs based photodetectors. The spray coating deposition method was particularly effective in this application due to the low volatility and large water surface tension and in particular for the low solute concentration. The work nicely showed how the chemical engineering of the QD compositions, size and surface chemistry by means of the proper choice of ligands, allowed reducing the trap states mostly localized at the QD surface. The presence of these trap states are considered to be responsible for the long response time of the photodetectors. Also a comparison between the excited states dynamics limiting the fast response of the photodetectors was provided. The author compared the faster response observed in $\mathrm{HgTe}-\mathrm{QDs}$ to the time response of PbSQDs photodetectors. They attributed such improvement to the faster bimolecular recombination and shorter lived trap states, which would enable the faster temporal response and high 


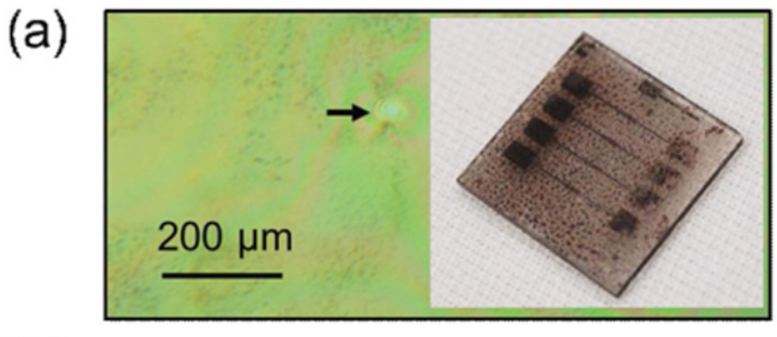

(b)
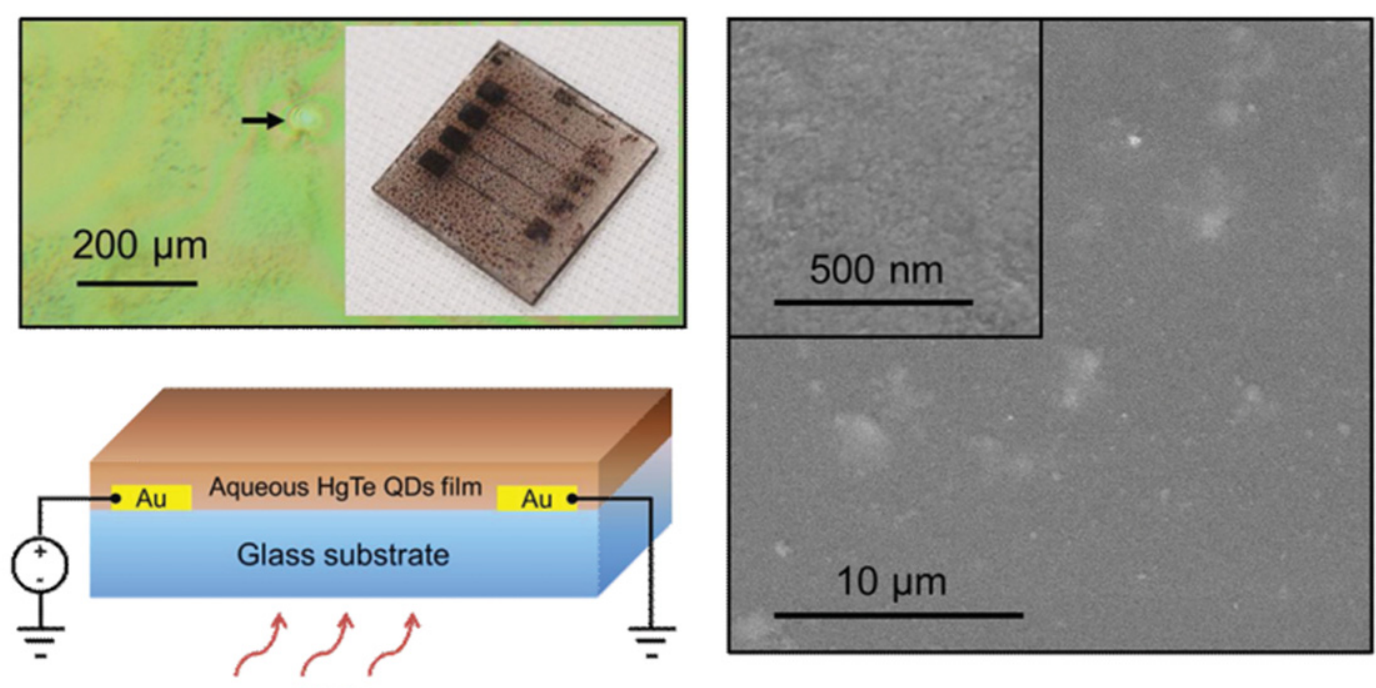

(c)

Liaht

Figure 14. (a)-(b) Device structure of a spray coated HgTe-QDs based photodetector. (c) Scanning electron microscopy images showing the surface morphology HgTe-QDs film [39]. (Reproduced with permission from [39] Copyright 2014, Wiley.)

photoconduction gain in the HgTe-QDs $\left(R=1100 \mathrm{~mA} \mathrm{~W}^{-1}\right.$ at $60 \mathrm{~V}$ and $1000 \mathrm{~nm} ; D^{*}=10^{10}$ Jones; $\mathrm{BW}=1 \mathrm{MHz}$ at $800 \mathrm{~mW} \mathrm{~cm}^{-2}$ ).

The examples reported in this section show that the advantages provided by spray coating rely mostly on the high production speed achievable and its compatibility with various substrates. Spray processing represents a good strategy to be employed when dealing with organic materials that have a poor solubility, which is the main limitation for many deposition techniques such as inkjet printing and spin coating.

In this section we have also seen that patterning with spray coating is obtained with the use of a shadow mask, resulting in a resolution limited to only tens of micrometers in the best case. Therefore the ease in applying such patterning process is counterbalanced by a limited lateral resolution, making it difficult to reach the high pattern resolution required by the most advanced and integrated technologies.

\section{Outlook}

Solution processable organic and inorganic semiconductors allow combining the advantages related to their peculiar physical chemistry with the ease of processability, enabling the preparation of printable inks and therefore the exploitation of printing technologies for the fabrication of large-area, printed, opto-electronic systems. The possibility to tailor their optical and electrical response by chemical design can give access to applications currently precluded or hardly addressable by standard inorganic detectors. The low production costs and the use of synthetic methods to produce a large amount of active materials, especially for the case of carbonbased inks, make printed photodetectors highly competitive. As an example, current inorganic electronics based on CMOS chips is generally still too expensive for disposable components.

Solution processed semiconductors can be printed on a wide variety of substrates, including ultra-thin substrates, fostering the development of conformable electronics. Therefore there is a consistent industrial effort to the development of printing technologies to produce low cost, flexible, light-weight photodetector arrays. New large area conformable organic image sensors on plastic and transparent backplanes, fabricated combining printed OPD with a plastic organic transistor backplane, have been already demonstrated (flexible sensor with a $4 \times 4 \mathrm{~cm}$ active area, $175 \mu \mathrm{m}$ pixel size, $375 \mu \mathrm{m}$ pitch and $94 \times 95=8930$ pixel resolu-tions) [76].

While printed photodetectors are comparable to the nonprinted ones, both organic and inorganic, in terms of responsivity and EQE, their operating speed does not exceed $1 \mathrm{MHz}$. These results point out that there is still more optimization to be done to reach at least the higher values reported for the organic non-printed counterpart [15-19].

Moreover, in most of printed OPDs works, $D^{*}$ was extrapolated by assuming that the dominant contribution to the noise was uniquely arising from the dark current shot noise. There are only limited reports of actual measurements of the real noise in photodetectors required for the proper estimation of their detectivity [23, 40, 69]. In particular flicker noise (frequency dependent noise) is likely to be dominant for low frequency applications and so this aspect should be investigated in a deeper way in order to make a more reliable characterization of the device performances.

The remaining issue still to be addressed is related to the uniformity of printed electronic devices and the yield of production over large areas. Another strict requirement for many applications, yet to be fulfilled, is the further downscaling of the device footprint to enable the integration into 
more complex electronic arrays. This challenging aspect is currently being investigated by the community. Also it is essential to assess and improve the stability of the printed materials, in particular their possible degradation upon exposure to ambient conditions. If such limitations are successfully overcome, in the near future we may witness the adoption of printed photodetectors in a large range of new applications such as digital image sensing [69], smart packaging and sensors for medical equipment and biomedical diagnostics [77], security and mobile electronics commerce.

\section{Acknowledgment}

This work was partially supported by Fondazione Cariplo through the InDiXi project grant n.2011-0368.

\section{Refereces}

[1] Logeeswaran V J et al 2011 IEEE J. Sel. Top. Quantum Electron. 171002

[2] Baeg K J, Caironi M and Noh Y Y 2013 Adv. Mater. 254210

[3] Wang X, Tian W, Liao M Y, Bando Y and Golberg D 2014 Chem. Soc. Rev. $\mathbf{4 3} 1400$

[4] Singh M, Haverinen H M, Dhagat P and Jabbour G E 2010 Adv. Mater. 22673

[5] Sondergaard R R, Hosel M and Krebs F C 2013 J. Polym. Sci. Pol. Phys. $\mathbf{5 1} 16$

[6] Krebs F C 2009 Sol. Energy Mater. Sol. C 93394

[7] Gong X, Tong M H, Xia Y J, Cai W Z, Moon J S, Cao Y, Yu G, Shieh C L, Nilsson B and Heeger A J 2009 Science 3251665

[8] Mishra A and Bauerle P 2012 Angew. Chem., Int. Ed. Engl. 512020

[9] Jiang W, Li Y and Wang Z H 2013 Chem. Soc. Rev. 426113

[10] Usta H, Facchetti A and Marks T J 2011 Acc. Chem. Res. 44 501

[11] Blakesley J C, Castro F A, Kylberg W, Dibb G F A, Arantes C, Valaski R, Cremona M, Kim J S and Kim J S 2014 Org. Electron. 151263

[12] Roncali J, Leriche P and Blanchard P 2014 Adv. Mater. 263821

[13] Altazin S, Clerc R, Gwoziecki R, Verilhac J M, Boudinet D, Pananakakis G, Ghibaudo G, Chartier I and Coppard R 2014 J. Appl. Phys. 115064509

[14] Peumans P, Bulović V and Forrest S R 2000 Appl. Phys. Lett. 763855

[15] Schilinsky P, Waldauf C, Hauch J and Brabec C J 2004 Thin Solid Films 451105

[16] Yang D Z, Zhou X K and Ma D G 2013 Org. Electron. 143019

[17] Zhu H L, Choy W C H, Sha W E I and Ren X G 2014 Adv. Opt. Mater. 21082

[18] Hamasaki T, Morimune T, Kajii H, Minakata S, Tsuruoka R, Nagamachi T and Ohmori Y 2009 Thin Solid Films 518548

[19] Zimmerman J D, Diev V V, Hanson K, Lunt R R, Yu E K, Thompson M E and Forrest S R 2010 Adv. Mater. 222780

[20] Park K S, Lee K S, Kang C M, Baek J, S H K, Lee C, Koo Lee Y E, Kang Y and Sung M M 2014 Nano Lett. 15289

[21] Konstantatos G and Sargent E H 2010 Nat. Nanotechnology 5391
[22] Kramer I J and Sargent E H 2014 Chem. Rev. 114863

[23] Boberl M, Kovalenko M V, Gamerith S, List E J W and Heiss W 2007 Adv. Mater. 193574

[24] Akhavan V A, Goodfellow B W, Panthani M G, Reid D K, Hellebusch D J, Adachi T and Korgel B A 2010 Energy Environ. Sci. 31600

[25] Rauch T, Boberl M, Tedde S F, Furst J, Kovalenko M V, Hesser G N, Lemmer U, Heiss W and Hayden O 2009 Nat. Photonics 3332

[26] Keuleyan S, Lhuillier E, Brajuskovic V and Guyot-Sionnest P 2011 Nat. Photonics 5489

[27] Lim A E J, Song J F, Fang Q, Li C, Tu X G, Duan N, Chen K K, Tern R P C and Liow T Y 2014 IEEE J. Sel. Top. Quantum Electron. 208300112

[28] Baeg K J, Binda M, Natali D, Caironi M and Noh Y Y 2013 Adv. Mater. 254267

[29] Cabanillas-Gonzalez J, Grancini G and Lanzani G 2011 Adv. Mater. 235468

[30] Lampert M A and Mark P 1970 Current Injection in Solids (Electrical Science) (New York: Academic)

[31] Kasap S O 2001 Optoelectronics and Photonics: Principles and Practices (Englewood Cliffs, NJ: Prentice-Hall)

[32] Donati S 1999 Photodetectors: Devices, Circuits and Applications (Englewood Cliffs, NJ : Prentice-Hall)

[33] Anthopoulos T D 2007 Appl. Phys. Lett. 91113513

[34] Wobkenberg P H, Labram J G, Swiecicki J-M, Parkhomenko $\mathrm{K}$, Sredojevic D, Gisselbrecht J-P, de Leeuw D M, Bradley D D C, Djukic J-P and Anthopoulos T D 2010 J. Mater. Chem. 203673

[35] Wojciechowski J R, Shriver-Lake L C, Yamaguchi M Y, Fureder E, Pieler R, Schamesberger M, Winder C, Prall H J, Sonnleitner M and Ligler F S 2009 Anal. Chem. 813455

[36] Azzellino G, Grimoldi A, Binda M, Caironi M, Natali D and Sampietro M 2013 Adv. Mater. 256829

[37] Wang H L, Cheng C, Zhang L, Liu H T, Zhao Y, Guo Y L, Hu W P, Yu G and Liu Y Q 2014 Adv. Mater. 264683

[38] Kim M, Ha H J, Yun H J, You I K, Baeg K J, Kim Y H and Ju B K 2014 Org. Electron. 152677

[39] Chen M Y, Yu H, Kershaw S V, Xu H H, Gupta S, Hetsch F, Rogach A L and Zhao N 2014 Adv. Funct. Mater. 2453

[40] Tedde S F, Kern J, Sterzl T, Furst J, Lugli P and Hayden O 2009 Nano Lett. 9980

[41] Inamdar S I and Rajpure K Y 2014 J. Alloy Compd. 59555

[42] Binda M, Natali D, Iacchetti A and Sampietro M $2014 A d v$. Mater. 254335

[43] Gili E, Caironi M and Sirringhaus H 2012 Inkjet Printing of Downscaled Organic Electronic Devices (New York: Wiley)

[44] Jung S, Sou A, Banger K, Ko D H, Chow P C Y, McNeill C R and Sirringhaus H 2014 Adv. Energy Mater. 4 1400432

[45] Lilliu S, Boberl M, Sramek M, Tedde S F, Macdonald J E and Hayden O 2011 Thin Solid Films 520610

[46] Lavery L L, Whiting G L and Arias A C 2011 Org. Electron. 12682

[47] Pace G, Grimoldi A, Natali D, Sampietro M, Coughlin J E, Bazan G C and Caironi M 2014 Adv. Mater. 266773

[48] Wu J D and Lin L Y 2014 IEEE Photonics Tech. Lett. 26737

[49] Yang Y, Omi S, Goto R, Yahiro M, Era M, Watanabe H and Oki Y 2011 Org. Electron. 12405

[50] Conibeer G, Willoughby A, Hoth C, Seemann A, Steim R, Ameri T, Azimi H and Brabec C J 2014 Solar Cell Materials: Developing Technologies ed. G Conibeer and A Willoughby (Chichester, UK: Wiley) p 217

[51] Manga K K, Wang S, Jaiswal M, Bao Q L and Loh K P 2010 Adv. Mater. 225265

[52] Finn D J, Lotya M, Cunningham G, Smith R J, McCloskey D, Donegan J F and Coleman J N 2014 J. Mater. Chem. C 2925 
[53] Kim Y-H, Han J-I, Han M-K, Anthony J E, Park J and Park S K 2010 Org. Electron. 111529

[54] Kim M, Koo J B, Baeg K J, Jung S W, Ju B K and You I K 2012 Appl. Phys. Lett. 10133306

[55] Mandal S, Dell'Erba G, Luzio A, Bucella S G, Perinot A, Calloni A, Berti G, Bussetti G, Duò L, Facchetti A, Noh Y Y and Caironi M 2015 Org. Electron. 20132

[56] Wu Y, Girgis E, Strom V, Voit W, Belova L and Rao K V 2011 Phys. Status Solidi A 208206

[57] Li J T, Naiini M M, Vaziri S, Lemme M C and Ostling M 2014 Adv. Funct. Mater 246524

[58] Katzir E, Yochelis S, Paltiel Y, Azoubel S, Shimoni A and Magdassi S 2014 Sensor Actuators B 196112

[59] Sebastian P J and Calixto M E 2000 Thin Solid Films 360128

[60] Sebastian P J and Ocampo M 1996 Sol. Energy Mat. Sol. C 441

[61] Olea A and Sebastian P J 1998 Sol. Energy Mat. Sol. C 55149

[62] Du H, Antonuk L E, El-Mohri Y, Zhao Q H, Su Z, Yamamoto J and Wang Y 2008 Phys. Med. Biol. 531325

[63] Schieber M, Zuck A, Gilboa H and Zentai G 2006 IEEE Trans. Nucl. Sci. 532385

[64] Cho S H, Heo S U, Choi C W, Kang S S, Nam S H, Park J K and Park S K 2008 J. Korean Phys. Soc. 521123

[65] Zhao Q H, Antonuk L E, El-Mohri Y, Wang Y, Du H, Sawant A, Su Z and Yamamoto J 2010 Med. Phys. 372738
[66] Kim S I, Kim S W, Park J C, Kim K, Han S W, Kim H K, Kim C J, U-In C, Yoo I-K and Kim K 2011 IEEE, Electron Devices Meeting p 14.2.1

[67] Park J C, Jeon P J, Kim J S and Im S 2015 Adv. Healthc. Mater. 451

[68] Tenent R C, Barnes T M, Bergeson J D, Ferguson A J, To B, Gedvilas L M, Heben M J and Blackburn J L $2009 A d v$. Mater. 213210

[69] Baierl D, Pancheri L, Schmidt M, Stoppa D, Dalla Betta G F, Scarpa G and Lugli P 2012 Nat. Commun. 31175

[70] Aga R, Jordan C, Aga R S, Bartsch C M and Heckman E M 2014 IEEE Electron Device Lett. 351124

[71] Liu Z, Khaled P, Li R, Dong R, Feng X and Müllen K 2014 Adv. Mater. 27669

[72] Kim S, Yim J, Wang X, Bradley D D C, Lee S and Demello J C 2010 Adv. Funct. Mater. 202310

[73] Falco A, Cina L, Scarpa G, Lugli P and Abdellah A 2014 ACS Appl. Mater. Interfaces 610593

[74] Schmidt M, Falco A, Loch M, Lugli P and Scarpa G 2014 AIP Adv. 4107132

[75] Shinde S S and Rajpure K Y 2012 J. Alloy. Compd. 522118

[76] http://isorg.fr/actu/2/plastic-logic-and-isorg-claim-theprestigious-flexi-award-for-their-revolutionary-flexibleplastic-image-sensor_206.htm

[77] Lochner C M, Khan Y, Pierre A and Arias A C 2014 Nat. Commun. 55745 